Pacific Journal of Mathematics

CHARACTERIZATION OF THE ENDOMORPHISM RINGS OF 


\title{
CHARACTERIZATION OF THE ENDOMORPHISM RINGS OF DIVISIBLE TORSION MODULES AND REDUCED COMPLETE TORSION- FREE MODULES OVER COMPLETE DISCRETE VALUATION RINGS
}

\section{WOLFGANG LIEBERT}

\begin{abstract}
Let $R$ be a complete discrete valuation ring (possibly non-commutative). If $K$ is the quotient field of $R$ then there is an isomorphism between the category of divisible torsion $R$-modules $G$ and the category of reduced complete torsionfree $R$-modules $H$ given by $G \rightarrow H=\operatorname{Hom}_{R}(K / R, G)$. Moreover, the $R$-endomorphism ring $E(G)$ is naturally isomorphic to the $R$-endomorphism ring $E(H)$ of $H$. It is the purpose of this paper to find necessary and sufficient conditions for an abstract ring to be isomorphic to the $R$-endomorphism ring of such an $R$-module.
\end{abstract}

Our problem has already been solved in the special case where $R$ is a (not necessarily commutative) field. In [16] Wolfson characterized the ring $E$ of all linear transformations of a vector space over a field by the following four properties: (1) $E_{0}$, the socle of $E$, is not a zero ring, and is contained in every nonzero two-sided ideal of $E$. (2) If $L$ is a left ideal of $E$ which is annihilated on the right only by zero, then $E_{0} \subset L$. (3) The sum of two left (right) annihilators is a left (right) annihilator. (4) $E$ possesses an identity element. Our main theorem may be considered as an extension of Wolfson's beautiful result to the case of an arbitrary complete discrete valuation ring. So, for example, in passing from the vector space case to this general one, "subspace" now becomes "direct summand" and "zero ideal" translates to "Jacobson radical". If $H$ is a vector space over a field $R$, then the structure of its $R$-endomorphism ring $E(H)$ is to a large extent determined by the ideal $E_{0}(H)$ of all $R$-endomorphisms which map $H$ onto a subspace of finite rank. In this case $E_{0}(H)$ is the socle of $E(H)$, the sum of all minimal left (right) ideals of $E(H)$. If $R$ is an arbitrary complete discrete valuation ring and $H$ a reduced complete torsion-free $R$-module, then $E_{0}(H)$ determines again the behavior of the entire ring $E(H)$. The proper generalization now reads: $E_{0}(H)$ is the sum of all minimal nonradical left (right) ideals of $E(H)$. Here we call an ideal of a ring $E$ nonradical if it is not contained in the Jacobson radical $J(E)$ of $E$. And by a minimal nonradical ideal we mean an ideal $I$ which is nonradical and has the property that every ideal of $E$ which is properly contained in $I$ belongs to $J(E)$. 
If we define the $J$-adic topology of a ring by taking the powers of its Jacobson radical as a neighborhood basis at zero, then we can state our main result as follows. The following three properties of an abstract ring $E$ are equivalent:

I. $E$ is isomorphic to the endomorphism ring of a divisible torsion module over a complete discrete valuation ring.

II. $E$ is isomorphic to the endomorphism ring of a reduced complete torsion-free module over a complete discrete valuation ring.

III. (1) $E$ is Hausdorff and complete in its $J$-adic topology.

(2) $J(E)=p E=E p$, where $p$ is either zero or a non-zero-divisor of $E$.

(3) $E_{0}$, the sum of all minimal nonradical right ideals of $E$, is not a zero ring, and is contained in every nonradical two-sided ideal of $E$.

(4) Let $L$ be a left ideal of $E$ which is closed in the $J$-adic topology of $E$ and satisfies $p E \cap L=p L$. If the right annihilator of $L$ is zero, then $L$ contains $E_{0}$.

(5) Let $L_{1}$ and $L_{2}$ be left annihilators in $E$ whose intersection is zero. If $p E \cap\left(L_{1}+L_{2}\right)=p\left(L_{1}+L_{2}\right)$, then $L_{1}+L_{2}$ is a left annihilator.

(6) $E$ has an identity element.

The similarity to Wolfson's characterization theorem is apparent. In fact, for $p=0$ these are Wolfson's conditions, however, our theorem doesn't say explicitly that in this case the complete discrete valuation ring reduces to a field. Our theorem is proved by modifying the methods used by Wolfson in [16]. The main tool is a Galois correspondence between the annihilators of the endomorphism rings and the direct summands of the underlying modules. Use is also made of the fact that these endomorphism rings are generated by their idempotents. This paper continues the author's work in $[10,11,12]$.

\section{Complete discrete valuation rings.}

Definition. A ring $R$ with Jacobson radical $J(R)$ is a complete discrete valuation ring if it satisfies the following conditions:

(1) $R / J(R)$ is a (not necessarily commutative) field.

(2) $J(R)=p R=R p$, where $p$ is either zero or a non-nilpotent element of $R$.

(3) $R$ is Hausdorff and complete in its $J$-adic topology.

(4) $R$ possesses an identity element.

Thus complete discrete valuation rings need not be commutative. 
A typical example is provided by the ring of all formal power series in one indeterminate $x$ over a (not necessarily commutative) field $F$ together with the rule $k x=x k^{\alpha}$ for all $x \in F$, where $\alpha$ is an automorphism of $F$.

Our intention is to give a ring-theoretical characterization of the endomorphism rings of certain $R$-modules. If $p=0$ in the definition above, then $R$ is a field and the $R$-modules are just the vector spaces. Since their endomorphism rings have already been characterized (Wolfson [16]), we shall henceforth assume that $p \neq 0$. The following properties of a complete discrete valuation ring $R$ are immediate consequences of the above definition. For more details see Baer [1, 3].

(2.1) $J(R)$ is the set of all non-units of $R$.

(2.2) Every nonzero ideal of $R$ is two-sided and has the form $J(R)^{i}=p^{i} R=R p^{i}$ with $0 \leqq i$.

(2.3) Every element $r \neq 0$ of $R$ has a unique representation of the form $r=p^{h(r)} s=t p^{h(r)}$, where $s$ and $t$ are units in $R$.

(2.4) $R$ has no zero-divisors.

(2.5) For every $t$ in $R$ there is a ring automorphism $\sigma(t)$ of $R$ with $r t=t r^{\sigma(t)}$ for all $r \in R$. For $t=0$ we define $\sigma(0)=1$. In particular $R$ satisfies the Ore condition

$$
x y=y x^{\sigma(y)}=y^{\sigma(x)-1} x
$$

for all $x, y \in R$.

(2.6) It is well known that (2.5) implies the existence of a unique quotient field $K$ of $R$ into which $R$ can be embedded. The elements of $K$ may be represented in the form $r p^{-i}$ with $r \in R$ and $0 \leqq i$. Addition and multiplication in $K$ are defined by

$$
\begin{aligned}
r p^{-i}+s p^{-j} & =\left(r p^{j}+s p^{i}\right) p^{-i-j} \\
\left(r p^{-i}\right) \cdot\left(s p^{-j}\right) & =\left(r s^{\sigma\left(p^{i}\right)}\right) p^{-i-j} .
\end{aligned}
$$

And the multiplicative inverse of $r p^{-i}$ is $\left(p^{i} t^{-1}\right) p^{-h(r)}$, if $r=p^{h(r)} t$ with $t$ a unit in $R$.

(2.7) Every ring automorphism $\alpha$ of $R$ can be extended to an automorphism $\alpha$ of $K$ by defining $\left(r p^{-i}\right)^{\alpha}=\left(r^{\alpha} \varepsilon_{i}^{-1}\right) p^{-i}$, where $\left(p^{i}\right)^{\alpha}=$ $p^{i} \varepsilon_{i}$ with $\varepsilon_{i}$ a unit in $R$. Then (2.5) yields that for every $r \in R$ there is an automorphism $\sigma(r)$ of $K$ which satisfies $k r=r k^{o(r)}$ for every $k \in K$. 
(2.8) The $R$-module $K / R$ contains for every $i$ exactly one submodule isomorphic to $R / p^{i} R$, and is the union of all of them. Therefore we have $R x=x R$ for every $x \in K / R$, that is to say, if $x \in K / R$ and $r \in R$ then there exists an element $s$ in $R$ such that $r x=x s$. Now only a minor modification of a proof in Fuchs [4] (Example 5, p. 211) is necessary to show that the $R$-endomorphism ring of $K / R$ is isomorphic to $R$. Moreover, it follows from (2.7) that for every $t \in R$ there exists a group automorphism $\sigma(t)$ of $K / R$ satisfying $(r x)^{\sigma(t)}=$ $r^{\sigma(t)} x^{\sigma(t)}$ and $x t=t x^{\sigma(t)}$ for all $r \in R$ and $x \in K / R$.

In the following $R$ will always be a complete discrete valuation ring with quotient field $K$. Once for all: module means left module and homomorphisms operate on the right side of their arguments. The ring of all $R$-endomorphisms of an $R$-module $M$ will be denoted by $E(M)$. We shall be concerned with divisible torsion and reduced complete torsion-free $R$-modules. The theory of these modules is almost identical with the theory of the corresponding modules over commutative valuation rings as presented in Kaplansky [7]. Only some slight formal changes have to be made in so far as typical commutative arguments will now involve the automorphisms $\sigma(r)$ described above. We shall briefly indicate some of these changes, others we leave to the reader.

3. Divisible torsion $R$-modules and their homomorphisms. An $R$-module $D$ is called divisible if $D=R D$. Alternatively, $D$ is divisible if $D=p D$. We call an $R$-module reduced if it has no nonzero divisible submodules. The following characterization of the divisible $R$-modules can be found in Baer [3] (§ 4) or Kaplansky [7] (§ 5).

THEOREM 3.1. The following properties of an $R$-module $D \neq 0$ are equivalent:

(1) $D$ is divisible.

(2) $D$ is a direct sum of modules each isomorphic to $K$ or $K / R$.

(3) $D$ is a direct summand of every containing module.

(4) If $M$ is an $R$-module and $N$ a submodule of $M$, then every $R$-homomorphism of $N$ into $D$ can be extended to an $R$-homomorphism of $M$ into $D$.

Let $M$ be an $R$-module. If $x$ is an element in $M$, then the order $o(x)$ of $x$ is the set of all $r \in R$ with $r x=0$. Evidently $o^{\prime}(x)$ is a left ideal in $R$. It follows from (2.2) that $o(x)$ is either zero or has the form $p^{i} R=R p^{i}$. The set of all $x$ in $M$ with $o(x) \neq 0$ is a submodule $t M$ of $M$, the so-called torsion submodule of $M$. If $t M=M$, we say that $M$ is a torsion module, and if $t M=0$, we shall say that $M$ is torsion-free. Now let $G$ be a divisible torsion $R$-module. We know 
from (3.1) that $G$ is the direct sum of modules each isomorphic to $K / R$. Define $G\left[p^{i}\right]=\left\{x \in G \mid p^{i} R \subset o(x)\right\}$. Then $G\left[p^{i}\right]$ is a submodule of $G$ which is fully invariant (it is sent into itself by every $R$-endomorphism of $G$ ). Moreover, $G\left[p^{i}\right]$ may be regarded in a natural way as an $R / p^{i} R$-module. We define the rank $\left.r_{(}^{\prime} G\right)$ of $G$ to be the rank of the vector space $G[p]$ over the field $R / p R$. Obviously $r(G)$ is just the cardinal number of $K / R$ summands in the above direct decomposition of $G$. It is a fact that $r(G)$ determines $G$ up to isomorphism as an $R$-module.

Now let $V(G)$ be the set of all divisible submodules of $G$. Then $V(G)$ is partially ordered by set-theoretical inclusion, and for any two elements $V_{1}, V_{2} \in V(G)$ there exists an inf $\left(V_{1}, V_{2}\right)$, namely the maximal divisible submodule of $V_{1} \cap V_{2}$, and a $\sup \left(V_{1}, V_{2}\right)$, namely $V_{1}+V_{2}$. Therefore $V(G)$ is a lattice, which is the same as the lattice of all direct summands of $G$. Let us briefly show how to recapture $r(G)$ from $V(G)$.

If $L$ is any lattice and $x$ an arbitrary element of $L$, then by an $x$-chain in $L$ we mean any subset $C$ of $L$ which is well-ordered by the lattice order such that each member of $C$ is bounded above by $x$ and is not a minimum of $L$. The rank of $x$ is the least upper bound of the set of all cardinals of $x$-chains in $L$.

Now consider $V(G)$ and pick $F \in V(G)$. Then $F$ is the direct sum of submodules $F_{i}, i$ ranging over an index set $I$, each $F_{i}$ isoinorphic with $K / R$. I can be well-ordered by some relation, say $>$. Let $S$ be the set of all nonempty $>$-initial segments of $I$. For each $T \in S$, let $F_{T}$ be the direct sum of the $F_{t}, t \in T$. Then the set of all $F_{T}$ for some $T \in S$ is a maximal $F$-chain in $V(G)$. It is immediate that every maximal $F$-chain may be obtained in this way and that every given $F$-chain can be refined to a maximal one. The cardinal number of each of these maximal $F$-chains is just $r(F)$. Hence the concept of lattice rank of $F$ coincides with its module rank.

For the following choose a fixed decomposition of $G$ into the direct sum of submodules $G_{i}$, each isomorphic to $K / R$. By (2.8), each $G_{i}$ possesses a group automorphism $\sigma_{i}(r)$ for every $r \in R$, satisfying $\left(\lambda g_{i}\right)^{\sigma_{i}(r)}=\lambda^{\sigma_{i}(r)} g_{i}^{\sigma_{i}(r)}$ for all $\lambda \in R$ and $g_{i} \in G_{i}$. We can therefore obtain a group automorphism $\sigma(r)$ of $G$ itself, satisfying $(\lambda g)^{\sigma(r)}=\lambda^{\sigma(r)} g^{\sigma(r)}$ for every $g \in G$, if we just define it componentwise: $\sigma(r)=\sigma_{i}(r)$ on $G_{i}$. Although $\sigma(r)$ need not be an $R$-automorphism, it maps submodules of $G$ onto submodules: if $S$ is a submodule, then $S^{\sigma(r)}=(R S)^{\sigma(r)}=$ $R^{\sigma(r)} S^{\sigma(r)}=R S^{\sigma(r)}$. And if $S$ is divisible and, say, $p^{\sigma(r)}=p r^{\prime}$ witl $r^{\prime}$ a unit in $R$, then $S^{\sigma(r)}=(p S)^{\sigma(r)}=p^{\sigma(r)} S^{\sigma(r)}=p r^{\prime} S^{\sigma(r)}=p S^{\sigma(r)}$, so that $S^{\sigma(r)}$ is divisible too. In particular, $\sigma(r)$ maps each $G\left[p^{i}\right]$ onto itself since for each $i \geqq 0, G\left[p^{i+1}\right] / G\left[p^{2}\right]$ is the sum of all minimal submodules of the $R$-module $G / G\left[p^{i}\right]$. 
Next, let $\lambda \in R$. Then (2.8) suggests that we define a map $\lambda$ of $G$ into itself by $g \lambda=\lambda g^{\sigma(\lambda)}$ for all $g \in G$. Clearly $\lambda$ is additive. Moreover, if $r \in R$, then

$$
(r g) \lambda=\lambda(r g)^{\sigma(\lambda)}=\lambda r^{\sigma(\lambda)} g^{\sigma(\lambda)}=r \lambda g^{\sigma(\lambda)}=r(g \lambda) .
$$

Consequently $\lambda$ is an $R$-endomorphism of $G$. If $\lambda$ belongs to the center of $R$, then $\sigma(\lambda)=1$, so that in this case the $R$-endomorphism $\lambda$ is just multiplication of the elements of $G$ by $\lambda$.

We have thus proved

LEMMA 3.2. For every $\lambda \in R, G$ possesses a group automorphism $\sigma(\lambda)$ and an $R$-endomorphism $\lambda$ satisfying

$$
\begin{aligned}
(r g)^{\sigma(\lambda)} & =r^{\sigma(\lambda)} g^{\sigma(\lambda)} & & \text { for all } r \in R \text { and } g \in G \\
g \lambda & =\lambda g^{\sigma(\lambda)} & & \text { for all } g \in G .
\end{aligned}
$$

Moreover, o $(\lambda)$ maps submodules onto submodules and divisible submodules onto divisible ones. In particular, each $G\left[p^{i}\right]$ is mapped onto itself by $\sigma(\lambda)$.

In the following let $F$ and $G$ be divisible torsion $R$-modules, and let us consider $H=\operatorname{Hom}_{R}(F, G)$, the set of all $R$-homomorphisms from $F$ into $G$. It follows from (3.2) that we can put an $R$-module structure on $H$ by defining $x(\lambda \phi)=\lambda x^{\sigma(\lambda)} \phi$ for all $x \in F, \lambda \in R$ and $\phi \in H$. Thus $\lambda \phi$ is the product of the $R$-endomorphism $\lambda$ of $F$ with the $R$-homomorphism $\phi$ from $F$ into $G$. But we emphasize that, unless $R$ is commutative or $F \cong K / R$, this is not a canonical way of turning $H$ into an $R$-module because in (3.2) the mappings $\lambda$ and $\sigma(\lambda)$ cannot be defined in a natural way. However, it will be a consequence of the following lemma that the submodules $p^{n} H$ of $H$ are independent of the particular choice of $\lambda$ and $\sigma(\lambda)$ in (3.2). Therefore we can introduce the $p$-adic topology on $H$ in a natural way by using the submodules $p^{n} H$ as neighborhoods of 0 . We wish to determine the $R$-module type of $H$.

Lemma 3.3. Let $\alpha \in H=\operatorname{Hom}_{R}(F, G)$. Then $\alpha$ annihilates $F\left[p^{n}\right]$ if and only if $\alpha \in p^{n} H$.

Proof. Assume $F\left[p^{n}\right] \alpha=0$ and let $f \in F$. Because $p^{n} F=F$, there exists an element $f^{\prime} \in F$ such that $f=p^{n} f^{\prime}$. Define a map $\beta$ of $F$ into $G$ by $f \beta=\left[f^{\sigma\left(p^{n}\right)-1}\right]^{\prime} \alpha$. Then $\beta$ is well-defined because if $p^{n} f_{1}=$ $p^{n} f_{2}$, then $f_{1}-f_{2} \in F\left[p^{n}\right]$, so that $f_{1} \alpha=f_{2} \alpha$. Clearly $\beta$ is additive. Let $r \in R$. Then $r f=r p^{n} f^{\prime}=p^{n} r^{\sigma\left(p^{n)}\right.} f^{\prime}$. Therefore we can choose $(r f)^{\prime}=r^{\sigma\left(p^{n)}\right.} f^{\prime}$. Thus 


$$
\begin{aligned}
(r f) \beta & =\left[(r f)^{\sigma\left(p^{n)-1}\right.}\right]^{\prime} \alpha=\left[r^{o\left(p^{n}\right)-1} f^{\sigma\left(p^{n)-1}\right.}\right]^{\prime} \alpha \\
& =\left(r\left[f^{\sigma\left(p^{n)-1}\right.}\right]^{\prime}\right) \alpha=r\left(\left[f^{\sigma\left(p^{n)-1}\right.}\right]^{\prime} \alpha\right)=r(f \beta) .
\end{aligned}
$$

Consequently $\beta$ is an $R$-homomorphism from $F$ into $G$. Also

$$
f p^{n} \beta=p^{n} f^{\sigma\left(p^{n}\right)} \beta=p^{n}\left(f^{\prime} \alpha\right)=\left(p^{n} f^{\prime}\right) \alpha=f \alpha .
$$

Therefore $\alpha=p^{n} \beta \in p^{n} H$.

Conversely, assume that $\alpha \in p^{n} H$, say, $\alpha=p^{n} \alpha^{\prime}$ for some $\alpha^{\prime} \in H$. Then from (3.2) we obtain

$$
F\left[p^{n}\right] \alpha=F\left[p^{n}\right]\left(p^{n} \alpha^{\prime}\right)=p^{n} F\left[p^{n}\right]^{\sigma\left(p^{n}\right)} \alpha^{\prime}=\left(p^{n} F\left[p^{n}\right]\right) \alpha^{\prime}=0 .
$$

This completes our proof.

Lemma 3.4. $\operatorname{Hom}_{R}(F, G)$ is a reduced torsion-free $R$-module which is Hausdorff and complete in its p-adic topology.

Proof. Let $H=\operatorname{Hom}_{R}(F, G)$. If $\alpha \in \bigcap_{n} p^{n} H$, then

$$
F \alpha=\left(\bigcup_{n} F\left[p^{n}\right]\right) \alpha=\bigcup_{n}\left(F\left[p^{n}\right] \alpha\right)=0 .
$$

Hence $\bigcap_{n} p^{n} H=0$. Consequently $H$ is reduced. Next, let $0 \neq \phi \in H$ and $\lambda \in o\left(\phi^{\prime}\right)=\{r \in R \mid r \dot{\phi}=0\}$. Assume $\lambda \neq 0$. Then $\lambda=\lambda^{\prime} p^{n}$ with $\lambda^{\prime}$ a unit in $R$ and $n \geqq 0$. Now, applying (3.2),

$$
0=F(\lambda \dot{\phi})=\left(\lambda F^{\sigma(\lambda)}\right) \dot{\phi}=(\lambda F) \dot{\phi}=\left(\lambda^{\prime} p^{n} F\right) \dot{\phi}=\left(\lambda^{\prime} F\right) \phi=F \dot{\phi} .
$$

But this contradicts our choice of $\phi$. Hence $o(\dot{\phi})=0$, so that $H$ is torsion-free.

Finally we establish the completeness of $H$ in its $p$-adic topology. It follows from $\bigcap_{n} p^{n} H=0$ that $H$ is a Hausdorff space. So let $\left(\alpha_{i}\right)$ be a Cauchy sequence in $H$. If $x \in F$, then $x \in F\left[p^{k}\right]$ for some positive integer $k$. By (3.3), there exists an index $j_{k}$ such that $\alpha_{i}-\alpha_{j_{k}}$ annihilates $F\left[p^{k}\right]$ for all $i \geqq j_{k^{\circ}}$. Hence $x x_{i}=x \alpha_{j_{k}}$ for all $i \geqq j_{k}$. Define $x \alpha=x \alpha_{j_{k}}$. Then $\alpha$ represents a well-defined $R$-homomorphism from $F$ into $G$. Moreover, $\alpha-\alpha_{i}$ annihilates $F\left[p^{k}\right]$ for all $i \geqq j_{k}$. By (3.3), this means that $\alpha-\alpha_{i} \in p^{k} H$. Thus $\alpha$ is the limit of $\left(\alpha_{i}\right)$. Consequently $H$ is complete.

We shall now turn to the special case $F=G$ and characterize the Jacobson radical of $E(G)=\operatorname{Hom}_{R}(G, G)$. Four further lemmas are needed. The first one follows immediately from (3.1) (4). Let $P\left(G\left[p^{n}\right]\right)$ denote the set of all $R$-endomorphisms of $G$ which annihilate $G\left[p^{n}\right]$. It is obvious that $P\left(G\left[p^{n}\right]\right)$ is a two-sided ideal of $E(G)$.

LEMMA 3.5. $E(G) / P\left(G\left[p^{n}\right]\right)$ is isomorphic with the $R / p^{n} R$-endomorphism ring of $G\left[p^{n}\right]$. 
LeMma 3.6. $P\left(G\left[p^{n}\right]\right)=p^{n} E(G)=E(G) p^{n}$.

Proof. The left hand equation is a special case of (3.3). To prove $p^{n} E(G) \subset E(G) p^{n}$, let $p^{n} \phi \in p^{n} E(G)$ and $g \in G$. Define $g \alpha=\left(g^{\sigma\left(p^{n}\right)} \phi\right)^{\sigma\left(p^{n}\right)-1}$. It is immediate that $\alpha$ is an additive map of $G$ into itself. If $r \in R$, then, by (3.2),

$$
\begin{aligned}
(r g) \alpha & =\left[(r g)^{\sigma\left(p^{n}\right)} \phi\right]^{\sigma\left(p^{n}\right)-1}=\left[r^{\sigma\left(p^{n}\right)} g^{\sigma\left(p^{n}\right)} \phi\right]^{\sigma\left(p^{n}\right)-1} \\
& =r\left[g^{\sigma\left(p^{n}\right)} \phi\right]^{\sigma\left(p^{n)}-1\right.}=r(g \alpha) .
\end{aligned}
$$

Thus $\alpha$ is an $R$-endomorphism of $G$. Moreover, again using (3.2),

$$
\begin{aligned}
g\left(\alpha p^{n}\right) & =(g \alpha) p^{n}=p^{n}(g \alpha)^{\sigma\left(p^{n}\right)}=p^{n}\left(g^{\sigma\left(p^{n}\right)} \dot{\phi}\right) \\
& =\left(p^{n} g^{\sigma\left(p^{n}\right)}\right) \dot{\phi}=\left(g p^{n}\right) \dot{\phi}=g\left(p^{n} \dot{\phi}\right) .
\end{aligned}
$$

Therefore $p^{n} \dot{\phi}=\alpha p^{n}$, and it follows that $p^{n} E(G) \subset E(G) p^{n}$.

We complete the proof by showing that $E(G) p^{n} \subset P\left(G\left[p^{n}\right]\right)$. If $\beta \in E(G)$, then, by (3.2),

$$
G\left[p^{n}\right] \beta p^{n}=p^{n} G\left[p^{n}\right]^{\sigma\left(p^{n}\right)} \beta=p^{n} G\left[p^{n}\right] \beta=0 .
$$

Lemma 3.7. If $0 \neq \beta \in R$, then the $R$-endomorphism $\beta$ of $G$ is not a zero-divisor in $E(G)$.

Proof. The fact that $\beta$ is not a left-zero-divisor in $E(G)$ follows from the torsion-freeness of $\operatorname{Hom}_{R}(G, G)$ in (3.4). On the other hand, if $\alpha \in E(G)$ and $\alpha \beta=0$, then we write $\beta=p^{i} \beta^{\prime}$ in $R$ with $\beta^{\prime}$ a unit in $R$ and obtain

$$
0=G \alpha \beta=(G \alpha) \beta=p^{i} \beta^{\prime}(G \alpha)^{\sigma(\beta)}=p^{i}(G \alpha)^{\sigma(\beta)} .
$$

But $G \alpha$, and therefore, by (3.2), also $(G \alpha)^{\sigma(\beta)}$ is divisible. Hence $(G \alpha)^{\sigma(\beta)}=0$, hence $G \alpha=0$, hence $\alpha=0$. Thus $\beta$ also cannot be a right-zero-divisor in $E(G)$.

Lemma 3.8. Let $\alpha \in E(G)$. Then $\alpha$ is an automorphism if and only if it induces an automorphism in $G[p]$.

Proof. The necessity is clear. Let $K(\alpha)$ be the kernel of $\alpha$. If $K(\alpha) \cap G[p]=0$, then $K(\alpha)=0$. Hence $\alpha$ is one-to-one. Moreover, $G \alpha$ is divisible since it is a homomorphic image of $G$. But $G[p] \subset$ $G \alpha$. Hence $G \alpha=G$, which completes the proof.

The first part of the following characterization of the Jacobson radical $J(E(G)$ ) of $E(G)$ also follows from a result of Utumi ([14], Lemma 8, p. 19). 
THEOREM 3.9. There exists a non-zero-divisor $p$ in $E(G)$ such that $J(E(G))=P(G[p])=p E(G)=E(G) p$.

Proof. The $R / p R$-endomorphism ring of the vector space $G[p]$ has zero Jacobson radical. Therefore (3.5) implies that $J(E(G)) \subset P(G[p])$. Conversely, let $\alpha \in P(G[p])$. Then it follows from (3.8) that $1-\alpha$ is a unit in $E(G)$. Since $P(G[p])$ is an ideal of $E(G)$, this implies that $\alpha \in J(E(G)$ ) (see Jacobson [6], pp. 9 and 10). Thus $P(G[p]) \subset J(E(G)$ ). Consequently $J(E(G))=P(G[p])$. The remainder is (3.6) and (3.7).

COROLlary 3.10. E(G) is Hausdorff and complete in its J-adic topology.

Proof. This is an immediate consequence of (3.4) and (3.9).

STRUCTURe THEOREM 3.11. The following properties of the divisible torsion $R$-modules $G_{1}$ and $G_{2}$ are equivalent:

(1) $E\left(G_{1}\right) \cong E\left(G_{2}\right)$

(2) $G_{1}[p] \cong G_{2}[p]$

(3) $G_{1} \cong G_{2}$

(4) $\quad V\left(G_{1}\right) \cong V\left(G_{2}\right)$

(5) $\quad r\left(G_{1}\right)=r\left(G_{2}\right)$

Proof. Let $E\left(G_{1}\right) \cong E\left(G_{2}\right)$. Then $E\left(G_{1}\right) / J\left(E\left(G_{1}\right)\right)$ and $E\left(G_{2}\right) / J\left(E\left(G_{2}\right)\right)$ are isomorphic. It follows from (3.5) and (3.9) that $E\left(G_{1}[p] \cong E\left(G_{2}[p]\right)\right.$. This implies $G_{1}[p] \cong G_{2}[p]$ (Baer [2], Structure Theorem, p. 183). It is now clear how to complete the proof.

4. Reduced complete torsion-free $R$-modules. Let $H$ be an $R$-module. If an element $y$ in $H$. is divisible by $p^{n}$ but not by $p^{n+1}$, then we say that $y$ has hiight $n$. And $y$ has infinite height if it is divisible by $p^{n}$ for every $n$. A submodule $B$ of $H$ is pure if $p^{n} B=$ $B \cap p^{n} H$ for all $n$, i.e., if its elements have the same height in $B$ as in $H$. In a torsion-free $R$-module the elements of infinite height form a divisible submodule. Consequently, if $H$ is reduced and torsion-free, then it is without elements of infinite height: $\bigcap_{n} p^{n} H=0$. We introduce the $p$-adic topology on such a module by using the submodules $p^{n} H$ as neighborhoods of 0 . If $H$ is complete in its $p$-adic topology, we shall simply call it complete. For the classification of the reduced complete torsion-free $R$-modules the notion of a basic submodule is essential. This concept has been introduced in the theory of abelian groups by Kulikov [8]. The following lemma is due to Warfield ([15], Lemma 3), where it is stated for commutative $R$. 
Lemma 4.1. Let $H$ be a torsion-free $R$-module and let $\phi: H \rightarrow H / p H$ be the natural epimorphism. Let $\left(x_{i}\right)$ be a $R / p R$-basis for $H / p H, i$ ranging over some index set $I$. Choose elements $y_{i}, i \in I$, in $H$ such that $y_{i} \phi=x_{i}$. Let $B$ be the submodule of $H$ generated by the $y_{i}$ 's. Then

(1) $B$ is a free $R$-module on the generators $y_{i}$.

(2) $H / B$ is divisible.

(3) $B$ is pure in $H$.

Definition. A submodule $B$ of a torsion-free $R$-module $H$ is called a basic submodule if it satisfies the conditions (1)-(3) of (4.1).

It is clear that every basic submodule of $H$ arises in this way. Since property (2) of (4.1) is equivalent with the density of $B$ in $H$, we obtain the following well-known characterization of the reduced complete torsion-free $R$-modules. (2) is essentially due to Kaplansky ([7], Theorem 22, p. 51) and (3) is analogous to results proved for p-groups by Leptin ([9], property 12, p. 85).

THEOREM 4.2. The following properties of a reduced torsion-free $R$-module $H$ are equivalent:

(1) $H$ is complete.

(2) If $B$ is a basic submodule of $H$, then $H$ is the completion (in the $p$-adic topology) of $B$.

(3) If $B$ is a basic submodule of $H$, then every $R$-homomorphism $B \rightarrow H$ extends uniquely to an $R$-endomorphism of $H$.

In (3.4) we saw that $H=\operatorname{Hom}_{R}(F, G)$ is a reduced complete torsion-free $R$-module, if $F$ and $G$ are divisible torsion $R$-modules. In the special case $F=K / R$ we shall now construct a basic submodule of $H$.

LEMMA 4.3. Let $G$ be a divisible torsion $R$-module and $H=$ $\operatorname{Hom}_{R}(K / R, G)$. Let $G=\bigoplus_{i \in I} G_{i}$ with each $G_{i} \cong K / R$. Choose isomorphisms $\dot{\phi}_{i}, i \in I$, between $K / R$ and $G_{i}$. Then the $\phi_{i}$ 's, considered as elements of $H$, generate a basic submodule of $H$.

Proof. By (4.1) we have to show that modulo $p H$ the $\phi_{i}$ 's form a basis of $H / p H$. To verify that they are linearly independent modulo $p H$, suppose that there exist finitely many $r_{j} \in R$ such that $\Sigma\left(r_{j} \phi_{j}\right)=$ $p \alpha$ with $\alpha \in H$. If $x \in K / R[p]$, then by (3.2) and (3.3)

$$
0=x p \alpha=x \Sigma\left(r_{j} \dot{\phi}_{j}\right)=\Sigma\left(x r_{j} \dot{\phi}_{j}\right)=\Sigma\left(r_{j} x^{\sigma\left(r_{j}\right)} \dot{\phi}_{j}\right) .
$$

But for arbitrary $y \in K / R$ the elements $y \dot{\phi}_{j}$ are independent in $G$ since $G$ is the direct sum of the $G_{i}{ }^{\prime} s$. This implies that all $r_{j} x^{\sigma\left(r_{j}\right)} \dot{\phi}_{j}=0$. 
Then $x\left(r_{j} \phi_{j}\right)=0$, whence $r_{j} \phi_{j} \in p H$, by (3.3). Therefore the $\phi_{i}^{\prime}$ 's are independent modulo $p H$. Assume now that $0 \neq \alpha \in H$. Then there exists $x \neq 0$ in $K / R$ such that $x \alpha \neq 0$. Write $x \alpha=\Sigma x_{i}$, with $x_{i} \in G_{i}$ (almost all $x_{i}=0$ ). It is clear that $x_{i}$ is contained in the cyclic submodule of $G_{i}$ which is generated by $x \phi_{i}$. Thus, we can find $t_{i} \in R$ such that $t_{i}\left(x \dot{\phi}_{i}\right)=x_{i}$. And from (2.8) we obtain the existence of $s_{i} \in R$ with $t_{i} x=x s_{i}$. It follows that

$$
x \Sigma\left(s_{i} \phi_{i}\right)=\Sigma x\left(s_{i} \phi_{i}\right)=\Sigma\left(x s_{i}\right) \phi_{i}=\Sigma\left(t_{i} x\right) \phi_{i}=\Sigma t_{i}\left(x \dot{\phi}_{i}\right)=\Sigma x_{i}=x \alpha .
$$

Hence, if $o(x)=p^{n} R$, then $x$ generates $K / R\left[p^{n}\right]$ and therefore $\alpha-$ $\Sigma\left(s_{i} \phi_{i}\right)$ annihilates $K / R\left[p^{n}\right]$. By (3.3), we have $\alpha-\Sigma\left(s_{i} \phi_{i}\right) \in p^{n} H$, so that the set $\left\{\phi_{i}, \alpha\right\}$ is no more independent. This proves that the $\dot{\phi}_{i}$ 's form a maximal modulo $p H$ linearly independent subset of $H$, as required.

Let $H$ be a reduced torsion-free $R$-module. We define the rank $r(H)$ of $H$ to be the vector space rank of $H / p H$ over $R / p R$. If $B$ is a basic submodule of $H$, then $B / p B \cong H / p H$. Therefore $r(H)=r(B)$. And $r(B)$ is of course equal to the usual rank of $B$ as a free $R$-module, that is, to the maximum number of linearly independent elements in $B$ (which determines $B$ up to isomorphism as an $R$-module). According to an analogous result for p-groups (Fuchs [4], Corollary 34.2, p. 115), two reduced complete torsion-free $R$-modules are isomorphic if and only if their basic submodules are isomorphic. Thus we obtain

LEMMA 4.4. The following properties of the reduced complete torsion-free $R$-modules $H_{1}$ and $H_{2}$ are equivalent:

(1) $H_{1} \cong H_{2}$

(2) $r\left(H_{1}\right)=r\left(H_{2}\right)$.

The next two lemmas shall be needed later. In the first one we determine the Frattini submodule $F(H)$ of $H$. It is defined to be the intersection of $H$ with all its maximal submodules. And the second direct summand lemma is again due to Kaplansky ([7], Theorem 23, p. 52).

\section{Lemma 4.5. If $H$ is an $R$-module, then $F(H)=p H$.}

Proof. If $H$ has no maximal submodules then $H$ is divisible (Baer [3], p. 51). In that case $F(H)=H=p H$. Next assume that $H$ possesses a maximal submodule $M$. Suppose $p H \notin M$. Then there is an element $h$ in $H$ such that $p h$ and $M$ generate $H$. Hence we can find elements $m \in M$ and $r \in R$ with $h=r p h+m$. Since $1-r p$ is a unit in $R$, we have $h=(1-r p)^{-1} m \in M$, a contradiction. We deduce that $p H \subset F(H)$. Finally $F(H / p H)=0$ implies $F(H)=p H$. 
Lemma 4.6. Let $H$ be a reduced torsion-free $R$-module and $S$ a pure complete submodule. Then $S$ is a direct summand of $H$.

Let $H$ be a reduced complete torsion-free $R$-module and $V(H)$ the set of all its pure complete submodules. The torsion-freeness of $H$ guarantees that the intersection of any number of pure submodules is again pure. Therefore $V(H)$ is a lattice with $\inf \left(S_{1}, S_{2}\right)=S_{1} \cap S_{2}$ and $\sup \left(S_{1}, S_{2}\right)$ equal to the unique smallest pure complete submodule of $H$ containing $S_{1}$ and $S_{2}$. Since the completion of a pure submodule is likewise pure in $H$ (Kaplansky [7], Lemma 20, p. 51), sup $\left(S_{1}, S_{2}\right)$ is the completion of the purification of $S_{1}+S_{2}$. By (4.6), $V(H)$ is the lattice of all direct summands of $H$. We shall have to say more about $V(H)$ in the following sections.

5. The Harrison-Matlis duality. Let $F$ and $G$ be divisible $R$ modules. We know from (3.4) that $\operatorname{Hom}_{R}(F, G)$ is a reduced complete torsion-free $R$-module. It is now easy to see that every reduced complete torsion-free $R$-module can be realized in this way as $\operatorname{Hom}_{R}(F, G)$ for suitable divisible torsion $R$-modules $F$ and $G$.

Lemma 5.1 Let $G$ be a divisible torsion $R$-module and $H=$ $\operatorname{Hom}_{R}(K / R, G)$. Then $r(H)=r(G)$.

Proof. Define $\phi_{i}, i \in I$, as in (4.3), so that they generate a basic submodule $B$ of $H$. Then the cardinality of the index set $I$ equals $r(B)$. But on the other hand, this cardinality is clearly just the rank $r(G)$ of $G$. Since $r(B)=r(H)$, it follows that $r(H)=r(G)$, as required.

Since, by (3.11) and (4.4), $G$ and $H$ are both determined up to isomorphism by their rank, we obtain

THEOREM 5.2. The mapping $G \rightarrow \operatorname{Hom}_{R}(K / R, G)$ gives a one-to-one correspondence between all divisible torsion $R$-modules $G$ and all reduced complete torsion-free $R$-modules.

This is one half of a duality exhibited by Harrison in [5] between the divisible torsion groups and the reduced complete torsion-free groups, and later generalized by Matlis in [13] for the corresponding modules over a (commutative) domain. The other half would be to establish the inverse correspondence between all reduced complete torsion-free $R$-modules $H$ and all divisible torsion $R$-modules, given by $H \rightarrow K / R \otimes_{R} H$. Again this may be done quite elementary (without using homological methods) by showing that the tensor product $K / R \otimes_{R} H$ is a direct sum of $r(H)$ copies of $K / R$ (see Fuchs [4], Theorem 65.4, p. 255). We leave it to the reader. 
We shall now exhibit natural isomorphisms between $V(G)$ and $V(H)$ and between $E(G)$ and $E(H)$, where $H=\operatorname{Hom}_{R}(K / R, G)$.

THEOREM 5.3. Let $G$ be a divisible torsion $R$-module and $H=$ $\operatorname{Hom}_{R}(K / R, G)$. For $U \in V(G)$ and $F \in V(H)$ define

$$
\begin{aligned}
& \phi: U \rightarrow \operatorname{Hom}_{R}(K / R, U) \\
& \gamma: F \rightarrow(K / R) F=\left\{x \alpha^{\prime} \mid x \in K / R, \alpha \in F\right\} .
\end{aligned}
$$

Then the mappings $\dot{\phi}$ and $\gamma$ are reciprocal isomorphisms between $V(G)$ and $V(H)$.

Proof. If $F$ is a submodule of $G$, then $\operatorname{Hom}_{R}(K / R, F)$ is naturally embedded in $\operatorname{Hom}_{R}(K / R, G)$. And if $G=G_{1} \oplus G_{2}$, then

$$
\operatorname{Hom}_{R}\left(K / R, G_{1} \oplus G_{2}\right)=\operatorname{Hom}_{R}\left(K / R, G_{1}\right) \oplus \operatorname{Hom}_{R}\left(K / R, G_{2}\right) \text {. }
$$

Therefore $\dot{\phi}$ maps direct summands of $G$ onto direct summands of $H$. Let $H=H_{1} \oplus H_{2}$ and define $G_{1}=(K / R) H_{1}=H_{1}^{r}$ and

$$
G_{2}=(K / R) H_{2}=H_{2}^{r} \text {. }
$$

We claim that $G=G_{1} \oplus G_{2}$. If $g \in G$, then there exists $k \in K / R$ and $\alpha \in H$ such that $k \alpha=g$. But $\alpha=\alpha_{1}+\alpha_{2}$ with $\alpha_{i} \in H_{i}$. Therefore $g=k \alpha=k\left(\alpha_{1}+\alpha_{2}\right)=k \alpha_{1}+k \alpha_{2} \in G_{1}+G_{2}$, which shows $G=G_{1}+G_{2}$. To prove that this sum is direct, we pick any $x \in G_{1} \cap G_{2}$. Then there exist elements $y_{i} \in K / R$ and $\beta_{i} \in H_{i}$ such that $x=y_{1} \beta_{1}=y_{2} \beta_{2}$. Without loss of generality we can assume $y_{1}=y_{2}=y$, since either $y_{1}=r y_{2}$ or $y_{2}=r y_{1}$ for some $r \in R$, so that by (2.8) we have, say, $y_{1} \beta_{1}=\left(r y_{2}\right) \beta_{1}=$ $\left(y_{2} s\right) \beta_{1}=y_{2}\left(s \beta_{1}\right)$ with $s \in R$ and $s \beta_{1} \in H$. Let $o(y)=p^{n} R$. Then $\{y\}=$ $K / R\left[p^{n}\right]$. Now, by (3.3), $y\left(\beta_{1}-\beta_{2}\right)=0$ implies that $\beta_{1}-\beta_{2}$ is divisible by $p^{n}$. This is possible only if $\beta_{1}$ and $\beta_{2}$ are both divisible by $p^{n}$. Then $x=y \beta_{1}=y \beta_{2}=0$. Hence $G=G_{1} \oplus G_{2}$. Therefore, in particular, $\gamma$ maps direct summands of $H$ onto direct summands of $G$.

If $U$ is a direct summand of $G$, then clearly $(K / R) \operatorname{Hom}_{R}(K / R, U)$ is contained in $U$. Conversely, let $u \in U$. If $o(u)=p^{m} R$, then pick an element $x$ in $K / R$ with $o(x)=p^{m} R$. By (3.1), the $R$-homomorphism from $\{x\}$ into $U$, which maps $x$ onto $u$, can be extended to an $R$ homomorphism of $K / R$ into $U$. So $U \subset(K / R) \operatorname{Hom}_{R}(K / R, U)$. Hence $U^{\dot{\phi} \gamma}=U$. Next, if $H=H_{1} \oplus H_{2}$, then clearly $H_{1}$ is contained in $\operatorname{Hom}_{R}\left(K / R,(K / R) H_{1}\right)$. Conversely, let $\alpha \in H$ with $(K / R) \alpha \subset(K / R) H_{1}$. We must show that this implies $\alpha \in H_{1}$. Suppose $\alpha \notin H_{1}$. Then $\alpha=$ $\alpha_{1}+\alpha_{2}$ with $\alpha_{1} \in H_{1}$ and $0 \neq \alpha_{2} \in H_{2}$. But, as above, $G=(K / R) H_{1} \oplus$ $(K / R) H_{2}$. Hence there exists $y \in K / R$ such that $0 \neq y \alpha_{2} \in(K / R) H_{2}$. Then $y \alpha=y \alpha_{1}+y \alpha_{2} \notin(K / R) H_{1}$, contradicting the choice of $\alpha$. So $\operatorname{Hom}_{R}\left(K / R,(K / R) H_{1}\right) \subset H_{1}$. Hence $H_{1}=H_{1}^{\gamma \phi}$. Thus $\phi$ and $\gamma$ are re- 
ciprocal correspondences between $V(G)$ and $V(H)$. This implies in particular that both mappings are one-to-one and exhaustive.

If $U \subset K$ are direct summands of $G$, then $U^{\phi} \subset K^{\phi}$; and if $M \subset N$ are direct summands of $H$, then $M^{r} \subset N^{r}$. Consequently $\phi$ and $\gamma$ are reciprocal isomorphisms between $V(G)$ and $V(H)$. This completes the proof.

THEOREM 5.4. Let $G$ be a divisible torsion $R$-module and $H=$ $\operatorname{Hom}_{R}(K / R, G)$. Then there is a natural isomorphism between $E(G)$ and $E(H)$.

Proof. There is a natural homomorphism $\alpha \rightarrow \alpha^{*}$ from $E(G)$ into $E(H)$, defined by $\zeta \alpha^{*}=\zeta \alpha$ for all $\zeta \in H$. Let $\zeta \beta=0$ for all $\zeta \in H$. Then $G \beta=(K / G) H \beta=0$, which implies $\beta=0$. Hence $\alpha \rightarrow \alpha^{*}$ is oneto-one. To prove that it is onto, let $\sigma \in E(H)$. We must find $\alpha \in E(G)$ such that $\alpha^{*}=\sigma$. Write $G=\bigoplus_{i \in I} G_{i}$ with $G_{i} \cong K / R$ and choose isomorphisms $\dot{\phi}_{i}$ from $K / R$ onto $G_{i}$. Considering the $\dot{\phi}_{i}$ 's as elements of $H$, define the $R$-endomorphism $\alpha$ of $G$ componentwise by $g_{i} \alpha=$ $\left(g_{i} \dot{\phi}_{i}^{-1}\right)\left(\dot{\phi}_{i} \sigma\right)$, if $g_{i} \in G_{i}$. Then obviously $\dot{\phi}_{i} \alpha^{*}=\phi_{i} \alpha=\phi_{i} \sigma$, so that $\alpha^{*}$ and $\sigma$ have the same effect on $\dot{\phi}_{i}$. But, by (4.3), the $\phi_{i}$ 's generate a basic submodule $B$ of $H$. Since $B$ is dense in $H$, it follows immediately that $\alpha^{*}=\sigma$, as required.

STRUCTURe THEOREM 5.5. The following properties of a divisible torsion $R$-module $G$ and a reduced complete torsion-free $R$-module $H$ are equivalent:

(1) $E(G) \cong E(H)$

(2) $H \cong \operatorname{Hom}_{R}(K / R, G)$

(3) $G \cong K / R \otimes_{R} H$

(4) $G[p] \cong H / p H$

(5) $V(G) \cong V(H)$

(6) $r(G)=r(H)$.

Proof. By (5.2) there exists a divisible torsion $R$-module $G_{1}$ such that $H \cong H_{1}=\operatorname{Hom}_{R}\left(K / R, G_{1}\right)$. Then, using (5.1), $r\left(G_{1}\right)=r\left(H_{1}\right)=$ $r(H)$. Also $E\left(G_{1}\right) \cong E\left(H_{1}\right) \cong E(H)$, by $(5.4)$, and $V\left(G_{1}\right) \cong V\left(H_{1}\right) \cong V(H)$, by (5.3).

Now, if (1) is true, then $E\left(G_{1}\right) \cong E(G)$. By (3.11), this implies that $r(G)=r\left(G_{1}\right)$. Thus $r(G)=r(H)$, so that (6) is a consequence of (1).

Suppose $r(G)=r(H)$. We know that $\operatorname{Hom}_{R}(K / R, G)$ has the same rank as $G$. Since, by (4.4), $H$ is determined up to isomorphism by its rank, (2) follows from (6).

If $H \cong H_{2}=\operatorname{Hom}_{R}(K / R, G)$, then clearly $V\left(H_{2}\right)$ is isomorphic with 
$V(H)$, and combining this isomorphism with the natural isomorphism between $V(G)$ and $V\left(H_{2}\right)$ of (5.3) yields an isomorphism of $V(G)$ onto $V(H)$, so that (5) is a consequence of (2).

If $V(G)$ is isomorphic with $V(H)$, then $V(G) \cong V\left(G_{1}\right)$. Hence, by (3.11), $E(G) \cong E\left(G_{1}\right)$. Consequently $E(G) \cong E(H)$. Thus (5) implies (1).

The equivalence of (4) and (6) is clear from our definition of $r(G)$, $r(H)$ resp. And (3) has only been included for the sake of completeness. This finishes the proof.

We can now obtain more information about a reduced complete torsion-free $R$-module $H$ and its $R$-endomorphism ring by dualizing the results of $\S 3$. We demonstrate the dualization of (3.9) and (3.10) which yields a characterization of the Jacobson radical $J(E(H))$ of $E(H)$. Let $\Lambda(p H)$ denote the set of all $R$-endomorphisms of $H$ which map $H$ into $p H$.

THEOREM 5.6. There exists a nonzero-divisor $p$ in $E(H)$ such that $J(E(H))=\Lambda(p H)=p E(H)=E(H) p$. And $E(H) / J(E(H))$ is isomorphic with the $R / p R$-endomorphism ring of $H / p H$. Moreover, $E(H)$ is Hausdorff and complete in its J-adic topology.

Proof. (5.2) implies the existence of a divisible torsion $R$-module $G$ such that $H \cong H_{1}=\operatorname{Hom}_{R}(K / R, G)$. By (5.4), there exists a natural isomorphism $*$ of $E(G)$ onto $E\left(H_{1}\right)$. Hence (3.9) implies $J\left(E\left(H_{1}\right)\right)=$ $P(G[p])^{*}=p^{*} E\left(H_{1}\right)=E\left(H_{1}\right) p^{*}$. We claim that $P(G[p])^{*}=\Lambda\left(p H_{1}\right)$. This is true because the following properties of $\alpha \in E(G)$ are equivalent:

$$
\alpha \in P(G[p]) ; G[p] \alpha=0 ;(K / R[p]) H_{1} \alpha^{*}=0 ; H_{1} \alpha^{*} \subset p H_{1} ; \alpha^{*} \in \Lambda\left(p H_{1}\right) .
$$

Here we have applied (3.1) (4), which yields $(K / R[p]) H_{1}=G[p]$, and also (3.3).

Now let $\beta$ be an isomorphism between $H_{1}$ and $H$. Then $\beta$ induces an isomorphism $\beta^{\prime}$ between $E\left(H_{1}\right)$ and $E(H)$, defined by $\phi \beta^{\prime}=\beta^{-1} \phi \beta$. It follows from (4.5) that $\left(p H_{1}\right) \beta=p H$. Therefore the following properties of the $R$-endomorphism $\phi$ of $H$ are equivalent:

$$
\dot{\phi} \in \Lambda\left(p H_{1}\right) ; H_{1} \dot{\phi} \subset p H_{1} ; H\left(\dot{\phi} \beta^{\prime}\right) \subset p H ; \dot{\phi} \beta^{\prime} \in \Lambda(p H) .
$$

Thus $\left[\Lambda\left(p H_{1}\right)\right] \beta^{\prime}=\Lambda(p H)$. Hence

$$
\begin{aligned}
J(E(H)) & =\left[J\left(E\left(H_{1}\right)\right)\right] \beta^{\prime}=\left[\Lambda\left(p H_{1}\right)\right] \beta^{\prime}=A(p H) \\
& =\left(p^{*} \beta^{\prime}\right) E(H)=E(H)\left(p^{*} \beta^{\prime}\right) .
\end{aligned}
$$

Now we change notation from $p^{*} \beta^{\prime}$ to $p$ and have the desired characterization of $J(E(H))$.

Finally we observe that the constructed isomorphism * $\beta$ ' between 
$E(G)$ and $E(H)$ induces an isomorphism between $E(G) / J(E(G))$ and $E(H) / J(E(H))$ and also, by (5.5), implies the existence of an isomorphism between $G[p]$ and $H / p H$. Therefore, applying (3.5) and (3.9), we obtain

$$
E(H) / J(E(H)) \cong E(G) / J(E(G)) \cong E(G[p]) \cong E(H / p H),
$$

as required. The remainder is immediate from (3.10).

We conclude this section with the following remark. In (5.6) we have defined $p \in E(H)$ to be the image of $p \in E(G)$ under an isomorphism between $E(G)$ and $E(H)$. Since $p \in E(G)$ in general cannot be defined in a natural way, the same is true for $p \in E(H)$. There is no way to avoid this if we allow $R$ to be noncommutative. Of course we can introduce $p \in E(H)$ directly (just as it is possible to prove (5.6) directly). This would go as follows. We choose a fix basic submodule $B=\bigoplus_{i \in I} B_{i}$ of $H$ with each $B_{i} \cong R^{+}$. Let $\lambda \in R$. By (2.5), there exists for each $i \in I$ a group automorphism $\sigma_{i}(\lambda)$ of $B_{i}$ satisfying $\left(r b_{i}\right)^{\sigma_{i}(\lambda)}=r^{\sigma_{i}(\lambda)} b_{i}^{\sigma_{i}(\lambda)}$ for all $r \in R$ and $b_{i} \in B_{i}$. This defines a group automorphism $\sigma(\lambda)$ of $B$ itself with $(r b)^{\sigma(\lambda)}=r^{\sigma(\lambda)} b^{\sigma(\lambda)}$ if we let $\sigma(\lambda)=$ $\sigma_{i}(\lambda)$ on $B_{i}$. Then we extend $\sigma(\lambda)$ to a unique group automorphism of $H$ satisfying $(r h)^{\sigma(\lambda)}=r^{\sigma(\lambda)} h^{\sigma(\lambda)}$. And now we can define an $R$-endomorphism $\lambda$ of $H$ by $h \lambda=\lambda h^{\sigma(\lambda)}$ for all $h \in H$. It is clear, however, that $\lambda \in E(H)$ not only depends on the decomposition of $B$ into the direct sum of the $B_{i}$ 's, but also on the choice of the isomorphisms between the $B_{i}$ 's and $R^{+}$.

6. A Galois theory. A right (left) annihilator of a ring $E$ is the totality of elements in $E$ which annihilate a certain subset of $E$ from the right (left). The intersection of any set of right (left) annihilators is again a right (left) annihilator. Therefore the set of all right (left) annihilators of $E$ forms a lattice. In this section we will relate the lattice of direct summands of a reduced complete torsionfree $R$-module $H$ to the lattice of the left (right) annihilators of its $R$-endomorphism ring $E(H)$. We closely follow methods by Bear in [2], where a Galois correspondence was developed between the subspaces of a vector space and the annihilators of its endomorphism ring. See also Wolfson [16].

Let $M$ be an $R$-module and $E(M)$ its $R$-endomorphism ring. Let $E$ denote any subring of $E(M)$. To each submodule $S$ of $M$ we associate two ideals of $E$, the right ideal $P(S)=\{\alpha \in E \mid S \alpha=0\}$ and the left ideal $\Lambda(S)=\{\beta \in E \mid M \beta \subset S\}$. If $T$ is a subset of $E$, then $K(T)$ denotes the kernel of $T$, i.e., the totality of elements $x$ in $M$ such that $x T=0$. And $R(T)$ denotes the totality of $\alpha$ in $E$ such that $T \alpha=0$. Similarly, $L(T)$ is the left annihilator of $T$. 
The proof of the following lemma may be found in Bear [2], Chapter V. 2 or in Wolfson [16], §2.

Lemma 6.1. Let $M$ be an $R$-module and $E$ any subring of $E(M)$.

(1) $\Lambda[K(T)]=L(T)$ for every subset $T$ of $E$.

(2) $P(M T)=R(T)$ for every subset $T$ of $E$.

(3) $P(S)=R[\Lambda(S)]$ for every submodule $S$ of $M$ satisfying $M A(S)=S$.

(4) $\Lambda(S)=L[P(S)]$ for every submodule $S$ of $M$ satisfying $K[P(S)]=S$.

In what follows, $H$ always is a reduced complete torsion-free $R$ module. We denote by $E_{0}(H)$ the set of all $R$-endomorphisms $\alpha$ of $H$ such that $H \alpha$ has finite rank. We shall simply call them finite $R$-endomorphisms of $H$. Clearly $E_{0}(H)$ is a two-sided ideal in $\left.E_{(}^{\prime} H\right)$. Many of the properties of $E(H)$ are already enjoyed by the subrings between $E_{0}(H)$ and $E(H)$. In fact, one can say that $E_{0}(H)$ determines the behavior of the entire ring $E(H)$.

Lemma 6.2. Let $E$ be a subring of $E(H)$ containing $E_{0}(H)$. Then $H \Lambda(S)=S=K[P(S)]$ holds for every direct summand $S$ of $H$.

Proof. Let $H=S \oplus Q$. Since $H \Lambda(S) \subset S$ follows from the definition of $\Lambda(S)$, it is only necessary to show that $S \subset H \Lambda(S)$. Write $S=S_{1} \oplus S_{2}$ with $r\left(S_{1}\right)=1$, and pick a generator $x$ of $S_{1}$. Then for every $s \in S$ there is an $R$-endomorphism $\alpha_{s}$ in $E_{0}(H)$ satisfying $x \alpha_{s}=s$ and $\left(S_{2}+Q\right) \alpha_{s}=0$. Now $H \alpha_{s}=R s \subset S$, and hence $\alpha_{s} \in \Lambda(S)$. Also $s \in H \alpha_{s} \subset H \Lambda(S)$, and therefore $S \subset H \Lambda(S)$. Thus $H \Lambda(S)=S$.

Now we show $K[P(S)]=S$. Clearly $S \subset K[P(S)]$. Suppose $h \in S$. Then $h=s+q$ with $s \in S$ and $0 \neq q \in Q$. Certainly we can find a finite $R$-endomorphism $\dot{\phi}$ of $H$ satisfying $S \dot{\phi}=0$ but $q \dot{\phi} \neq 0$. Hence $0 \neq h \phi \in h P(S)$, which implies $h \notin K[P(S)]$, so that $K[P(S)] \subset S$, completing the proof.

Lemma 6.3. If $\alpha \in E(H)$, then $K(\alpha)$ is a direct summand of $H$.

Proof. Suppose that $y \in K(\alpha)$ is a multiple of $p^{n}$ in $H$, say $y=$ $p^{n} h$. Then $0=y \alpha=\left(p^{n} h\right) \alpha=p^{n}(h \alpha)$, so that $h \alpha=0$ by the torsionfreeness of $H$. Thus $y$ is already a multiple of $p^{n}$ in $K(\alpha)$. Hence $K(\alpha)$ is a pure submodule of $H$. Moreover, we claim that it is complete. Let $\left(x_{i}\right)$ be a Cauchy sequence in $K(\alpha)$. Then $\left(x_{i}\right)$ has a limit $x$ in $H$. For any given integer $n>0$, we have that for large $i, x-x_{i}$ lies in $p^{n} H$. Since $p^{n} H$ is a fully invariant submodule of $H$, this 
implies $x \alpha \in \bigcap_{n} p^{n} H=0$. Hence $x$ is already the desired limit in $K(\alpha)$ of the sequence $\left(x_{i}\right)$. Therefore, by $(4.6), K(\alpha)$ is a direct summand of $H$.

Lemma 6.4. Let $E$ be a subring of $E(H)$ which contains $E_{0}(H)$.

(1) If $A$ is a right annihilator, then $K(A)$ is a direct summand of $H$ and $A=P[K(A)]$.

(2) If $Q$ is a left annihilator, then $H Q$ is a direct summand of $H$ and $Q=\Lambda(H Q)$.

Proof. Let $A$ be a right annihilator, so that $A=R(T)$ for some subset $T$ of $E$. Then $R(T)=P(H T)$, by (6.1). Let $D$ be the smallest direct summand of $H$ containing $H T$. Then it follows from (6.3) that $P(H T)=P(D) . \quad$ Now $\quad K(A)=K[P(H T)]=K[P(D)]=D$, by $\quad(6.2)$. Hence $A=P[K(A)]$.

If $Q$ is a left annihilator, then $Q=L(S)$ for some subset $S$ of $E$. But $L(S)=\Lambda[K(S)]$, by (6.1). The intersection of any number of direct summands of $H$ is a direct summand of $H$. Since $K(S)=$ $\bigcap_{\beta \in S} K(\beta)$, we obtain from (6.3) that $K(S)$ is a direct summand of $H$. Therefore, by (6.2), $H Q=H \Lambda[K(S)]=K(S)$. This completes the proof.

We can now proceed as in Baer [2] and Wolfson [16] to obtain the following fundamental result.

THEOREM 6.5. Let $E$ be a subring of $E(H)$ containing all finite $R$-endomorphisms of $H$.

(1) The mappings $S \rightarrow P(S)$ and $A \rightarrow K(A)$ are reciprocal lattice anti-isomorphisms between the lattice of all direct summands $S$ of $H$ and the lattice of all right annihilators $A$ of $E$.

(2) The mappings $S \rightarrow \Lambda(S)$ and $Q \rightarrow H Q$ are reciprocal lattice isomorphisms between the lattice of all direct summands $S$ of $H$ and the lattice of all left annihilators $Q$ of $E$.

It is well-known (Baer [2], Proposition 3, p. 179) that in the endomorphism ring of a vector space over a field the sum of two left (right) annihilators is again a left (right) annihilator. This is no longer true for $E(H)$. Suppose $H$ has rank two, say $H=\{x\} \oplus\{y\}$. Then $L_{1}=\Lambda(\{x\})$ and $L_{2}=\Lambda(\{x+p y\})$ are left annihilators (with intersection zero), but their sum $L_{1}+L_{2}=\Lambda(\{x\} \oplus\{p y\})$ is not. Therefore in general, the smallest left (right) annihilator containing two given left (right) annihilators of $E(H)$ will be larger than the sum of the two. 
THEOREM 6.6. Suppose that $L_{1}$ and $L_{2}$ are left annihilators in $E(H)$ with $L_{1} \cap L_{2}=0$. If $\left(L_{1}+L_{2}\right) \cap p E(H)=p\left(L_{1}+L_{2}\right)$, then $L_{1}+$ $L_{2}$ is a left annihilator.

Proof. It is an immediate consequence of (6.5) that there exist direct summands $S_{1}$ and $S_{2}$ of $H$ with $L_{1}=\Lambda\left(S_{1}\right)$ and $L_{2}=\Lambda\left(S_{2}\right)$ and $S_{1} \cap S_{2}=0$. We wish to show that $S_{1}+S_{2}$ is a direct summand of $H$ and that $L_{1}+L_{2}=\Lambda\left(S_{1}+S_{2}\right)$. Let $p^{n} x=s_{1}+s_{2} \in S_{1}+S_{2}$ with $x \notin p H$. Then $H=\{x\} \oplus M$ for a suitable submodule $M$. Let $\sigma$ be the projection of $H$ onto $\{x\}$ along $M$. There exist $\alpha_{i} \in L_{i}$ with $x \alpha_{i}=s_{i}$. Let $\beta$ be an $R$-endomorphism of $H$ with $x \beta=p^{n} x$. Then $\sigma \beta \sigma=\sigma\left(\alpha_{1}+\right.$ $\left.\alpha_{2}\right) \in L_{1}+L_{2}$. Also $\sigma \beta \sigma \in \Lambda\left(p^{n} H\right)$. By (5.6), there exists $\phi$ in $E(H)$ such that $\sigma \beta \sigma=p \phi \sigma$. Since, by hypothesis, $L_{1}+L_{2}$ is "pure" in $E(H)$, it follows that $\phi \sigma \in L_{1}+L_{2} \subset \Lambda\left(S_{1}+S_{2}\right)$. Now write $x p=$ $p r x+p y$ with $y \in M$ are $r \in R$. Let $y \dot{\phi} \sigma=t x$ and $x \dot{\phi} \sigma=s x$ with $s, t \in R$. Then

$$
p^{n} x=x \beta=x p \dot{\phi} \sigma=p r x \dot{\phi} \sigma+p y \phi \sigma=p r s x+p t x=p(r s+t) x,
$$

so that $r s+t=p^{n-1}$. Therefore $t$ and $s$ cannot both be contained in $p^{n} R$. Since $t x$ and $s x$ are both contained in $S_{1}+S_{2}$, we conclude that $p^{n-1} x \in S_{1}+S_{2}$. By induction, $x$ itself is an element of $S_{1}+S_{2}$. Therefore $S_{1}+S_{2}$ is pure in $H$.

Next, let $\left(z_{i}\right)$ be a Cauchy sequence in $S_{1}+S_{2}$ whose limit in $H$ is $z$. Write $z_{i}=s_{i 1}+s_{i 2}$ with $s_{i 1} \in S_{1}$ and $s_{i 2} \in S_{2}$. Then the purity of $S_{1}+S_{2}$ together with $S_{1} \cap S_{2}=0$ imply that $\left(s_{i 1}\right)$ and $\left(s_{i 2}\right)$ are Cauchy sequences in $S_{1}, S_{2}$ resp. Since $S_{1}$ and $S_{2}$ are both complete, they have limits $z_{1}$ and $z_{2}$ in $S_{1}, S_{2}$ resp. Then of course we must have $z=z_{1}+$ $z_{2} \in S_{1}+S_{2}$, which shows that $S_{1}+S_{2}$ is complete. Thus $S_{1}+S_{2}$ is a direct summand of $H$, so that $\Lambda\left(S_{1}+S_{2}\right)$ is a left annihilator in $E(H)$, by (6.5). If $\dot{\phi}$ is any element in $A\left(S_{1}+S_{2}\right)$ and $h$ any element in $H$, then $h \dot{\phi}$ is an element in the direct sum $S_{1} \oplus S_{2}$. Hence there exist uniquely determined elements $h_{1}$ and $h_{2}$ in $S_{1}$ and $S_{2}$ resp. such that $h \dot{\phi}=h_{1}+h_{2}$. Define $\phi_{i}$ by $h \dot{\phi}_{i}=h_{i}$. Then $\dot{\phi}_{i} \in \Lambda\left(S_{i}\right)$ and $\dot{\phi}=\dot{\phi}_{1}+\dot{\phi}_{2}$. Hence $L_{1}+L_{2}=\Lambda\left(S_{1}+S_{2}\right)$.

7. The finite endomorphisms. Throughout this section we assume that $H$ is a reduced complete torsion-free $R$-module. We have called an $R$-endomorphism $\alpha$ of $H$ finite if the submodule $H \alpha$ of $H$ has finite rank. The main purpose of this section is to characterize the ideal $E_{0}(H)$ of all finite $R$-endomorphisms of $H$ inside the ring $E(H)$. We require four lemmas.

We shall term an ideal of a ring $E$ nonradical, if it is not contained in the Jacobson radical $J(E)$ of $E$. And by a minimal non- 
radical left (right) ideal of $E$ we mean a left (right) ideal $L$ which is nonradical and has the property that every proper subideal of $L$ is contained in $J(E)$.

LEMMA 7.1. Let $E$ be a ring with Jacobson radical $J(E)$. Suppose that $E$ contains a non-zero-divisor $p$ such that $J(E)=p E=E p$ and $\bigcap_{n} p^{n} E=0$. Then every minimal nonradical right (left) ideal of $E$ is a principal ideal generated by an idempotent.

Proof. We shall only prove the statement for right ideals since the left ideal case is similar.

Let $K$ be a minimal nonradical right ideal of $E$. If $k^{2} \in J(E)$ for all $k \in K$, then $[K+J(E)] / J(E)$ would be nil and consequently $K \subset$ $J(E)$. Therefore we can find an element $k$ in $K$ such that $k^{2} \notin J(E)$. The minimality of $K$ implies $K=k K=k^{2} E$.

Now suppose that for some $\alpha \in E$ we have $\alpha p \in \bar{K}$. We shall show that this implies $\alpha \in K$. We can write $\alpha p=k^{2} \beta$ for a suitable $\beta \in E$. Suppose that $k \beta \notin J(E)$. Then $k \beta E=K$, by the minimality of $K$. Therefore there exists a $\gamma \in E$ such that $k \beta \gamma=k$. Hence $k^{2}=k^{2} \beta \gamma=$ $\alpha p \gamma \in J(E)$. But this would contradict our choice of $k$. If follows that $k \beta \in J(E)$. Thus we can find an element $\phi$ in $E$ such that $k \beta=\phi p$. Now $\alpha p=k^{2} \beta=k(\dot{\phi} p)=(k \dot{\phi}) p$, so that $(\alpha-k \dot{\phi}) p=0$. Since $p$ is not a zero-divisor, we must have $\alpha=k \phi$, which implies that $\alpha \in K$. It now follows from the hypothesis $\bigcap_{n} p^{n} E=0$ that every nonzero element of $K$ has a unique representation of the form $x p^{n}$ with $n \geqq 0$ and $x \in K$ but $x \notin J(E)$.

Next let us consider $S=R(k) \cap K$, the set of all elements in $K$ which annihilate $k$ from the right. Suppose $S \neq 0$. Let $s$ be a nonzero element of $S$. We can write $s=s^{\prime} p^{m}$ with $m \geqq 0$ and $s^{\prime} \in K$ but $s^{\prime} \notin J(E)$. Then $s^{\prime} E=K$. Now pick $\sigma \in E$ with $s^{\prime} \sigma=k$ and write $p^{m} \sigma^{\prime}=\sigma p^{m}$. Then

$$
0=0 \sigma^{\prime}=(k s) \sigma^{\prime}=k s^{\prime} p^{m} \sigma^{\prime}=k s^{\prime} \sigma p^{m}=k^{2} p^{m},
$$

which yields $k^{2}=0$, since $p$ is not a zero-divisor. This contradicts again our choice of $k$. Hence $S=0$.

Finally, we observe that $k K=K$. Therefore we can find a nonzero element $e \in K$ such that $k e=k$. Then $e^{2}-e \in S$. Thus the element $e$ is the desired idempotent in $K$. We now have $K=e E$ since $K$ is minimal nonradical and $e \notin J(E)$. The proof is complete.

A nonzero idempotent of a ring is called minimal if it cannot be written as the sum of two orthogonal idempotents. Obviously, the 
minimal idempotents of $E(H)$ are just the projections of $H$ onto direct summands of rank one. Thus, if $e$ is a minimal idempotent in $E(H)$, then there is a ring isomorphism $e E(H) e \cong R$.

Lemma 7.2. The following properties of a left [right] ideal $X$ of $E(H)$ are equivalent:

(1) There exists a direct summand $F$ of rark [co-rank] one in $H$ such that $X=\Lambda(F)[X=P(F)]$.

(2) $X$ is a principal left [right] ideal gonsrated by a minimal idempotent.

(3) $X$ is a minimal left [right] annihilator.

(4) $X$ is a minimal nonradical left [right] ideal.

Proof. The equivalence of (1), (2) and (3) is an immediate consequence of (6.5). We complete the proof by showing that (2) and (4) are equivalent. Let $X$ be a principal left [right] ideal of the form $E(H) e[e E(H)]$, with $e$ a minimal idempotent of $E(H)$. Let $Y$ be a left [right] ideal of $E(H)$ which is properly contained in $X$. Then $e \notin Y^{2}$. Furthermore, $Y^{2}=Y e Y e[e Y e Y]$. Therefore $e Y e \neq$ $e E(H) e$. But $e E(H) e \cong R$. Hence $e Y e \subset J(e Y e)=e Y e \cap J(E(H))$. This implies $Y^{2} \subset J(E(H))$, whence $Y \subset J(E(H))$, since $E(H) / J(E(H))$ has no nonzero nilpotent ideals. Accordingly, $X$ is minimal nonradical, so that (4) is a consequence of (2). Finally, the implication (4) $\rightarrow(2)$ follows from (7.1) and (5.6).

Lemma 7.3. If $Z_{1}$ and $Z_{2}$ are inonradical two-sided ideals of $E(H)$, then $Z_{1} Z_{2}$ is likewise nonradical.

Proof. If $x \in H$ and $x \in p H$, and if $y \in H$, then there exists an $R$-endomorphism of $H$ mapping $x$ onto $y$. By (5.5), $J(E(H))=\Lambda(p H)$. Therefore the hypothesis implies the existence of an element $h \in H$ such that $h \notin p H$ and $h Z_{1}=H$. If $Z_{1} Z_{2} \subset \Lambda(p H)$, then $H Z_{2}=h Z_{1} Z_{2}=$ $p H$. Hence $Z_{2} \subset \Lambda(p H)$, which is a contradiction.

Lemma 7.4. $J\left(E_{0}(H)\right)=p E_{0}(H)=E_{0}(H) p$, and $E_{0}(H) / J\left(E_{0}(H)\right)$ is isomorphic with $E_{0}(H / p H)$, the ring of all finite $R / p R$-endomcrphisms of $H / p H$.

Proof. Let $\alpha p \in E_{0}(H)$. Then, by (6.3), $H=H_{1} \oplus K(\alpha p)$ with $r\left(H_{1}\right)$ finite. But $0=K(\alpha p) \alpha p=[K(\alpha p) \alpha] p$ implies that $K(\alpha p)=K(\alpha)$, since $K(p)=0$ follows from (6.3) and the fact that $p$ is not a zerodivisor in $E(H)$. Hence $H \alpha=H_{1} \alpha$, hence $r(H \alpha)$ is finite. Thus $\alpha \in$ $E_{0}(H)$.

Next, if $\dot{\phi}$ is any element of $E(H)$, then by (5.6) there exists an 
element $\phi^{\prime}$ in $E(H)$ such that $p \dot{\phi}=\phi^{\prime} p$. Since $p$ is not a zero-divisor in $E(H)$, the mapping $\dot{\phi} \rightarrow \phi^{\prime}$ constitutes an automorphism of $E(H)$. As a consequence of the following theorem, $E_{0}(H)$ is automorphism invariant. Therefore, if $p \alpha \in E_{0}(H)$, then $\alpha^{\prime} p \in E_{0}(H)$, hence $\alpha^{\prime} \in E_{0}(H)$, hence $\alpha \in E_{0}(H)$.

Since $E_{0}(H)$ is an ideal, we now obtain from (5.6) that $J\left(E_{0}(H)\right)=$ $E_{0}(H) \cap J(E(H))=p E_{0}(H)=E_{0}(H) p$.

To prove the statement about $E_{0}(H) / J\left(E_{0}(H)\right)$, note that $p H$ is a fully invariant submodule of $H$. Therefore every $\alpha \in E_{0}(H)$ induces a finite $R / p R$-endomorphism $\alpha^{*}$ in $H / p H$. The map $\alpha \rightarrow \alpha^{*}$ is a ring homomorphism from $E_{0}(H)$ into $E_{0}(H / p H)$ with kernel

$$
\Lambda(p H) \cap E_{0}(H)=J\left(E_{0}(H)\right) .
$$

We claim that it is an epimorphism. So let $\dot{\phi} \in E_{0}(H / p H)$, and let $\eta, \rho$ be the natural maps $H \rightarrow H / p H, R \rightarrow R / p R$ resp. We may pick a $R / p R$-basis $\left(x_{i}\right), i \in I$, for $H / p H$ such that $x_{i} \dot{\phi}=0$ for almost all $i \in I$. Next, we choose elements $y_{i}, i \in I$, in $H$ such that $y_{i} \eta=x_{i}$. Then, by (4.1), the submodule $B$ of $H$, generated by the $y_{i}$ 's, is a basic submodule of $H$. And we can write $x_{i} \dot{\phi}=\sum_{j_{\in} I}\left(r_{i j} x_{j}\right)$ with $r_{i j} \in R / p R$ and $r_{i j}=0$ for almost all $j$. Then choose elements $s_{i j}$ in $R$ such that $s_{i j} \rho=r_{i j}$ and $s_{i j}=0$ if $r_{i j}=0$. Finally, define $y_{i} \beta=$ $\sum_{j \in I}\left(s_{i j} y_{j}\right)$. Then $\beta$ is a finite $R$-endomorphism of $B$, and by (4.2), it can be extended to a unique $R$-endomorphism $\gamma$ of $H$. Since $B \gamma$ is a basic submodule of $H \gamma$, we have $(B \gamma) / p B \gamma=(H \gamma) / p H \gamma$, so that $r(H \gamma)$ must be finite. Consequently $\gamma \in E_{0}(H)$, and by construction we have $\gamma^{*}=\phi$. Thus $\alpha \rightarrow \alpha^{*}$ is onto. Hence $E_{0}(H) / J\left(E_{0}(H)\right) \cong$ $E_{0}(H / p H)$, as required.

We are now ready for the characterization of $E_{0}(H)$.

THEOREM 7.5. $E_{0}(H)$ is both, the sum of all minimal nonradical right ideals and the sum of all minimal nonradical left ideals of $E(H)$. Considered as a ring, $E_{0}(H)$ is simple modulo its Jacobson radical. Moreover, $E_{0}(H)$ is contained in every nonradical two-sided ideal of $E(H)$.

Proof. If $\alpha$ is a finite $R$-endomorphism of $H$, then by (6.3) we can find a submodule $F$ of finite rank such that $H=F \oplus I(\alpha)$. Since $F$ is free, the first assertion of the theorem follows from (7.2).

It is well-known (Jacobson [6], Structure Theorem, p. 75) that the finite endomorphisms of a vector space over a field form a simple ring. Therefore (7.4) implies that $E_{0}(H) / J\left(E_{0}(H)\right)$ is simple.

Finally, let $Z$ be a nonradical two-sided ideal of $E(H)$. Then 
$Z E_{0}(H)$ is nonradical too, by (7.3). Hence $E_{0}(H)=Z E_{0}(H) \subset Z$. This completes the proof.

The following result shows among other things how we can recapture the $R$-module $H$ from its $R$-endomorphism ring $E(H)$.

THEOREM 7.6. Suppose that $M=e E(H)$ is a minimal nonradical right ideal of $E(H)$ [e is a minimal idempotent of $E(H)$ ]. Let $R$ and $e E(H) e$ be endowed with their J-adic topologies. Then there is a topological isomorphism $\beta$ of the $R$-module $H$ upon the $e E(H) e$-module $M$ which satisfies $(H L)^{\beta}=M \cap L$ for every left ideal $L$ of $E(H)$.

Proof. We have $H=H e \oplus K(e)$, where $H e=R x$ has rank one. To every element $r \in R$ there is one and only one $R$-endomorphism $r^{\beta}=e r^{\beta} e$ of $H$ with the property $x r^{\beta}=r x$ and $K(e) r^{\beta}=0$. And to every element $h \in H$ there exists one and only one $R$-endomorphism $h^{\beta}=e h^{\beta}$ of $H$ with the properties $x h^{\beta}=h$ and $K(e) h^{\beta}=0$. It is easy to check that $\beta$ effects a topological module isomorphism between the $R$-module $H$ and the $e E(H) e$-module $M$.

Let $L$ be a left ideal of $E(H)$ and $b \in H L$. Then $b=y \lambda$ with $y \in H$ and $\lambda \in L$. There exists an $\alpha$ in $M$ such that $x \alpha=y$. Since $M$ is a right and $L$ a left ideal, we have $\alpha \lambda \in M \cap L$. It follows that $b^{\beta}=(y \lambda)^{\beta}=(x \alpha \chi \lambda)^{\beta}=\alpha \lambda$, so that $(H L)^{\beta} \subset M \cap L$. Conversely, pick $\dot{\phi} \in M \cap L$. Then $\dot{\phi}=(x \dot{\phi})^{\beta} \in(H L)^{\beta}$, which implies that $M \cap L \subset(H L)^{\beta}$. Thus $(H L)^{\beta}=M \cap L$.

COROLLARY 7.7. If $L$ is a left ideal of $E(H)$, then $H L$ is a submodule of $H$.

We conclude this section with an important property of the finite endomorphisms of $H$.

THEOREM 7.8. Let $L$ bo a left ideal of $E(H)$ which is closed in the $J$-adic topology of $E(H)$ and satisfies $L \cap p E(H)=p L$. Then $R(L)=0$ if and only if $L$ contains $E_{0}(H)$.

Proof. $H L$ is a submodule of $H$, by (7.7). We claim that it is pure. Let $h \in H$ and $p h \in H L$, so that $p h=h^{\prime} \lambda$ with $\lambda \in L$. Choose a decomposition $H=\{x\} \oplus F$ of $H$ and let $\pi$ be the projection of $H$ onto $\{x\}$ along $F$. If $\dot{p} \in \mathbb{E}(H)$ is such that $x \dot{\rho}=h^{\prime}$, then $x \pi \dot{\rho} \lambda=p h$ and $\pi \phi \lambda \in \pi E(H) \cap L$. We derive from (5.6) [or from (7.6)] that $\pi \phi \lambda \in p E(H)$. Hence $\pi \phi \lambda \in p L$, say, $\pi \dot{\phi} \lambda=p \alpha$ with $\alpha \in L$. Now $p h=$ $x p \alpha=p x^{\prime} \alpha$ for suitable $x^{\prime} \in H$, which implies that $h=x^{\prime} \alpha \in H L$. This shows that $H L$ is a pure submodule of $H$. 
Mioreover, $H L$ is complete. To see that, let $\left(x_{i}\right)$ be a Cauchy sequence in $H L$, say, $x_{i}=y_{i} \lambda_{i}$ with $\lambda_{i} \in L$. Now $\pi E(H)$ is a minimal nonradical right ideal of $E(H)$, and there exist $\beta_{i} \in \pi E(H)$ such that $x \beta_{i}=y_{i}$. Then $x\left(\beta_{i} \lambda_{i}\right)=x_{i}$ and $\beta_{i} \lambda_{i} \in \pi E(H) \cap L$. Now (7.6) shows that $\left(\beta_{i} \lambda_{i}\right)$ is a Cauchy sequence in the $\pi E(H) \pi$-module $\pi E(H)$. But $J[\pi E(H) \pi]=\pi E(H) \pi \cap J(E(H))$. Therefore $\left(\beta_{i} \lambda_{i}\right)$ is Cauchy in $E(H)$. Since $\beta_{i} \lambda_{i} \in L$, and since $L$ is closed in the $J$-adic topology of $E(H)$, this sequence has a limit $\lambda$ in $L$. Evidently then $\left(x_{i}\right)$ converges to $x \lambda$ in $H L$. Thus $H L$ is a pure complete submodule of $H$, and as such it is a direct summand, by (4.6).

Suppose that $R(L)=0$. By $(6.1), R(L)=P(H L)$. Hence $P(H L)=0$. But this implies that $H L=H$, since $H L$ is a direct summand. If now $M$ is any minimal nonradical right ideal of $E(H)$, then, by (7.6), $M=H^{\beta}=(H L)^{\beta}=M \cap L$. Accordingly, every minimal nonradical right ideal of $E(H)$ is contained in $L$, so that $E_{0}(H) \subset L$ follows from (7.5). Conversely, if $E_{0}(H) \subset L$, then clearly $H L=H$. Applying(6.1), we see that $0=P(H)=P(H L)=R(L)$, as was to be proved.

8. The idempotents. In this section we establish the very useful fact that the $R$-endomorphism ring of a reduced complete torsion-free $R$-module $H$ is generated by its idempotents. For the proof we shall need the following argument. If $\boldsymbol{H}_{\nu}$ is any cardinal number smaller than or equal to $r(H)$, then there exists a decomposition $H=$ $H_{1} \oplus H_{2}$ of $H$ with $r\left(H_{1}\right)=\aleph_{\nu}$. To see that, choose a basic submodule $B$ of $H$. Then $r(H)=r(B)$, and certainly we can decompose $B$ into a direct sum $B_{1} \oplus B_{2}$ with $r\left(B_{1}\right)=H_{\nu}$. If $H_{1}$ and $H_{2}$ denote the $p$-adic completions of $B_{1}$ and $B_{2}$ resp., then $H=H_{1} \oplus H_{2}$ and $r\left(H_{1}\right)=r\left(B_{1}\right)$.

Lemma 8.1. Suppose that $H=M \oplus N$ with $r(N) \geqq r(M) \geqq 1$. Let $\phi \in E(H)$ satisfy $N \dot{\phi}=0$. Then $\phi$ belongs to the subring of $E(H)$ generated by all idempotents of $E(H)$.

Proof. Let $\pi$ be the projection of $H$ onto $M$ along $N$. Because of $r(N) \geqq r(M)$, we can write $N=N^{\prime} \oplus N^{\prime \prime}$ with $r\left(N^{\prime}\right)=r(M)$. Let $\sigma$ be an isomorphism between $M$ and $N^{\prime}$. Then there exist $R$-endomorphisms $\alpha, \beta$ and $\gamma$ of $H$, defined as follows:

$$
\begin{array}{lllll}
\alpha=0 & \text { on } M & \alpha=1 & \text { on } N^{\prime} & \alpha=0 \text { on } N^{\prime \prime} \\
\beta=1 & \text { on } M & \beta=\sigma^{-1} \dot{\phi} \pi & \text { on } N^{\prime} & \beta=0 \text { on } N^{\prime \prime} \\
\gamma=\phi(1-\pi) & \text { on } M & \gamma=1 & \text { on } N^{\prime} & \gamma=1 \text { on } N^{\prime \prime} .
\end{array}
$$

Then $\alpha^{2}=\alpha, \beta^{2}=\beta$ and $\gamma^{2}=\gamma$. Finally, let $\tau=\pi \alpha \beta$ and $\rho=\pi \gamma$. Then for all $x \in M$,

$$
\begin{aligned}
& x \tau=x \pi \alpha \beta=x \alpha \beta=x \sigma \beta=x \sigma \sigma^{-1} \dot{\phi} \pi=x \dot{\phi} \pi \\
& x \rho=x \pi \gamma=x \gamma=x \dot{\phi}(1-\pi)
\end{aligned}
$$


and

$$
\begin{aligned}
& N \tau=(N \pi) \alpha \beta=0=N \phi=N \phi \pi \\
& N \rho=(N \pi) \gamma=0=N \dot{\phi}=N \phi(1-\pi) .
\end{aligned}
$$

Hence $\tau=\phi \pi$ and $\rho=\phi(1-\pi)$, and therefore $\phi=\tau+\rho$. This completes the proof of the lemma, since $\tau$ and $\rho$ are generated by the idempotents $\pi, \alpha, \beta$ and $\gamma$.

THEOREM 8.2. The ring $E(H)$ is generated by its idempotents if and only if the rank of $H$ is at least two.

Proof. If $r(H)=1$, then $E(H)=R$; and the only idempotents of $R$ are 0 and 1 which clearly do not generate $R$ as a ring (since we are assuming throughout this paper that $R$ is not a field).

Let $r(H) \geqq 2$. If $r(H)$ is infinite, then we break up $H=H_{1} \oplus H_{2}$ with $r\left(H_{1}\right)=r\left(H_{2}\right)$. For every $\beta$ in $E(H)$ we have $\beta=\varepsilon \beta+(1-\varepsilon) \beta$, where $\varepsilon$ is the projection of $H$ onto $H_{1}$ along $H_{2}$. Applying (8.1), we see that $\varepsilon \beta$ as well as $(1-\varepsilon) \beta$ are generated by idempotents in $E(H)$.

If $r(H)$ is finite and even, then again we only have to write $H=$ $H_{1} \oplus H_{2}$ with $r\left(H_{1}\right)=r\left(H_{2}\right)$ and apply the lemma. Suppose finally that $r(H)=2 n+1$ with $n \geqq 1$. Then $H=M \oplus N$ with $r(N)=1$. If $w$ denotes the projection of $H$ onto $M$ along $N$, then for each $\alpha \in E(H)$ we may write

$$
\alpha=w \alpha+(1-w) \alpha=w \alpha w+w \alpha(1-w)+(1-w) \alpha .
$$

Then (8.1) tells us that $(1-w) \alpha$ is generated by idempotents. The same is true for $w \alpha w$, since it is an $R$-endomorphism of the module $H w=M$, whose rank is even. Therefore we only must show that $w \alpha(1-w)$ is generated by idempotents. This, however, follows from $w \alpha(1-w)=[w+w \alpha(1-w)]-w$, where $w$ and $w+w \alpha(1-w)$ are both idempotent. This completes the proof.

9. The characterization theorem. In this last section we shall characterize the $R$-endomorphism rings of the divisible torsion $R$ modules and the reduced complete torsion-free $R$-modules. First, a preparatory lemma which generalizes a well-known fact for semisimple rings.

Lemma 9.1. Let $E$ be a ring and $0 \neq e=e^{2} \in E$. Then the following three statements are equivalent:

(1) $e E$ is a minimal nonradical right ideal

(2) $e E e / J(e E e)$ is a field

(3) Ee is a minimal nonradical left ideal.

Proof. Assume that $e E$ is minimal nonradical. Let $\alpha \in e E e$, but 
$\alpha \notin J(e E e)$. Then $\alpha \notin J(E)$, since $J(e E e)=e E e \cap J(E)$. But $\alpha \in e E$. Hence $\alpha e E=e E$. Thus $\alpha e E e=e E e$, which shows that $e E e / J(e E e)$ is without proper nonzero right ideals. It is well-known that this is equivalent with (2). Conversely, assume the validity of (2). Let $R$ be a right ideal of $E$ which is properly contained in $e E$. Then $R e=$ $e R e$ is a proper right ideal of $e E e$ because it doesn't contain $e$. Hence $e R e \subset J(e E e)$, hence $e R e \subset J(E)$, hence $R^{2}=e R e R \subset J(E)$, hence $R \subset$ $J(E)$. This proves the equivalence of (1) and (2). By symmetry, (2) and (3) are equivalent.

If a ring is without minimal nonradical right ideals, then we define the sum of its minimal nonradical right ideals to be the zero ideal.

MAIN THeOREm 9.2. Let $E$ be a ring and $E_{0}$ the sum of its minimal nonradical right ideals. Then the following three properties are equivalent:

I. There exists one and essentially only one complete discrete valuation ring $S$ and one and essentially only one divisible torsion $S$-module $G$ such that $E$ is isomorphic to the ring of all S-endomorphisms of $G$.

II. There exists one and essentially only one complete discrete valuation ring $R$ and one and essentially only one reduced complete torsion-free $R$-module $H$ such that $E$ is isomorphic to the ring of all $R$-endomorphisms of $H$.

III. (1) $E$ is Hausdorff and complete in its J-adic topology.

(2) There exists a non-zero-divisor $q$ in $E$ such that $J(E)=$ $q E=E q$.

(3) $E_{0}$ is not a zero ring and is contained in every nonradical two-sided ideal of $E$.

(4) Let $L$ be a left ideal of $E$ which is closed in the J-adic topology of $E$ and satisfies $q E \cap L=q L$. If $R(L)=0$ then $L$ contains $E_{0}$.

(5) If $L_{1}$ and $L_{2}$ are left annihilators in $E$ whose intersection is zero, and if $q E \cap\left(L_{1}+L_{2}\right)=q\left(L_{1}+L_{2}\right)$, then $L_{1}+L_{2}$ is a left annihilator.

(6) E possesses an identity element.

Proof. The equivalence of I and II is a consequence of (5.5). It remains to show that II and III are equivalent. Assume that $E \cong$ $E(H)$, where $H$ is a reduced complete torsion-free $R$-module. Then (1) and (2) of III follows from (5.6). Furthermore, (3) holds by virtue of (7.5), and (4) is true by (7.8). Finally, (5) is satisfied by (6.6), and clearly $E(H)$ possesses an identity element. Hence II implies III. 
Assume now that (1)-(6) of III are valid. The proof, that this implies the existence of one and essentially only one reduced complete torsion-free $R$-module $H$ such that $E \cong E(H)$, will be given in ten steps.

(a) Construction of the $R$-module $H$ such that $E$ is essentially a subring of $E(H)$. By (3), there exists a minimal nonradical right ideal $H$ in $E$. And from (1) and (7.1) it follows that there exists a minimal idempotent $e$ in $E$ such that $H=e E$. The right annihilator $R(H)$ of $H$ is a two-sided ideal of $E$. Assume that $0 \neq \alpha \in R(H)$. It is a consequence of (1) and (2) that for every element in $E$ there is a maximal power of $q$ dividing it. So let $\alpha=\beta q^{n}$ with $\beta \notin J(E)$. Since $q$ is not a zero-divisor, we must have $\beta \in R(H)$ so that $R(H) \not \subset$ $J(E)$. Then $E_{0} \subset R(H)$, by (3). This implies $H^{2} \subset H E_{0}=0$, which contradicts $H^{2}=H$. Hence $R(H)=0$, and $H$ is a faithful right $E$ module. The centralizer of $H=e E$ is the ring $R=e E e$ (Jacobson [6], Proposition 3, p. 51). We may therefore identify $E$ with a subring of the $R$-endomorphism ring $E(H)$ of the left $R$-module $H$.

(b) If $n$ is a positive integer then $I_{n}=\left\{\phi \in E \mid H \phi \subset J(E)^{n}\right\}=$ $J(E)^{n}=q^{n} E=E q^{n}$. The $I_{n}$ 's are two-sided ideals in $E$. From (2) it is clear that $J(E)^{n}=q^{n} E=E q^{n}$. And obviously $J(E)^{n} \subset I_{n}$. We prove the opposite inclusion by induction on $n$. If $I_{1} \not \subset J(E)$ then $E_{0} \subset I_{1}$, by (3). However, this would imply $H=H^{2} \subset H E_{0} \subset J(E)$, contradicting the choice of the ideal $H$ as a nonradical one. Hence $I_{1} \subset J(E)$. Next, assume that $I_{n} \subset J(E)^{n}$ and let $c \in I_{n+1}$. Then, since $I_{n+1} \subset I_{n}$, we can write $c=c^{\prime} q^{n}$. If $h \in H$, then $h c^{\prime} q^{n}=h c \in q^{n+1} E$. Thus $c^{\prime} \in I_{1}$ since $q$ is not a zero-divisor. Therefore $c \in q^{n+1} E$. Consequently $I_{n+1} \subset$ $J(E)^{n+1}$.

(c) There exists a non-zero-divisor $p$ in the ring $e E e$ such that $e E \cap J(E)^{n}=p^{n} e E, E e \cap J(E)^{n}=E e p^{n}$ and $e E e \cap J(E)^{n}=p^{n} e E e=e E e p^{n}$ for every positive integer $n$. Suppose that $e J(E) e \subset J(E)^{2}$. Then it follows from (b) that $q e \in J(E)^{2}=q^{2} E$. But then $e \in J(E)$, since $q$ is not a zero-divisor. The Jacobson radical of a ring, however, doesn't contain nonzero idempotents. Hence $e J(E) e \not \subset J(E)^{2}$. Now pick any $\alpha$ in $E$ such that $p=e \alpha q e \notin J(E)^{2}$ and write $q e=e^{\prime} q$. Note that $e^{\prime}$ is an idempotent. Then $\beta=e \alpha e^{\prime} \notin J(E)$. Therefore $\beta E=e E$ since $e E$ is minimal nonradical. If $e d q$ is arbitrary in $e E \cap J(E)$, then there exists $f \in E$ with $\beta f=e d$. Write $f q=q f^{\prime}$. Then

$$
e d q=\beta f q=\beta q f^{\prime}=e \alpha e^{\prime} q f^{\prime}=e \alpha q e f^{\prime}=p f^{\prime}=p e f^{\prime} .
$$

Thus $e E \cap J(E)$ is a principal right ideal in $E$ generated by $p$. We know from (9.1) that $E e$ is a minimal nonradical left ideal in $E$. Therefore, by symmetry, $E e \cap J(E)$ is a principal left ideal generated by $p$. An easy induction argument now asserts that the first two wanted equations hold for all $n$. Then the validity of the third one 
is established by

$$
\begin{aligned}
p^{n} e E e & =p^{n} e E \cap E e=\left[e E \cap J(E)^{n}\right] \cap E e=e E e \cap J(E)^{n} \\
& =\left[E e \cap J(E)^{n}\right] \cap e E=E e p^{n} \cap e E=e E e p^{n} .
\end{aligned}
$$

Next, we claim that neither in $e E$ nor in $E e$ is $p$ a zero-divisor. Suppose $\phi p=0$ for $\phi \in E e$. Then $\phi \beta q=0$ implies that $\phi \beta=0$, hence $\phi e E=0$ since $\beta E=e E$. Thus $\phi \in E(1-e) \cap E e=0$, whence $\dot{\phi}=0$. By symmetry, also $p \phi=0$ with $\phi \in e E$ implies $\dot{\phi}=0$.

(d) $J(e E e)^{n}=e E e \cap J(E)^{n}$. Since $J(e E e)=e E e \cap J(E)$ is true in any ring it follows from (c) that $J(e E e)=p e E e=e E e p$. Hence $J(e E e)^{n}=p^{n} e E e$ which in turn equals $e E e \cap J(E)^{n}=p^{n} e E e$ which in turn equals $e E e \cap J(E)^{n}$, by (c).

(e) $R=e E e$ is a complete discrete valuation ring. First, we have that $R / J(R)$ is a field, by (9.1). Secondly, we know from (c) that $J(R)=p R=R p$, with $p$ a non-zero-divisor in $R$. Of course $p$ is not nilpotent. Moreover, if $\left\{\alpha_{i}\right\}$ is a Cauchy sequence in the $J$-adic topology of $E$ which is contained in $e E e$, then $\lim \alpha_{i}=\lim \left(e \alpha_{i} e\right)=$ $e\left(\lim \alpha_{i}\right) e \in e E e$. Hence $e E e$ is a closed subspace of $E$. Therefore the fact that $R$ is Hausdorff and complete in its $J$-adic topology is an immediate consequence of (1), if we just observe that, by (d), the $J$-adic topology on $R$ is the same as its relative topology induced by the $J$-adic topology of $E$.

(f) $H=e E$ is a reduced complete torsion-free $R$-module. $H$ is torsion-free since we know from (c) that $p$ is not a zero-divisor. And $H$ is reduced because $\bigcap_{n} p^{n} H \subset \bigcap_{n} q^{n} E=0$. Now endow $H$ with its $p$-adic topology by taking the submodules $p^{i} H$ for $i \geqq 0$ as a neighborhood basis at zero. Then it follows from (c) that this topology coincides with the relative topology on $H$ induced by the $J$-adic topology of $E$. Since $e E$ is a closed subspace of $E$, we conclude that $H$ is complete in its $p$-adic topology.

Recall that we have identified $E$ with a subring of $E(H)$. In the following we shall use the operators $\Lambda$ and $P$ as defined in $\S 6$.

(g) $H \Lambda(S)=S$ for every submodule $S$ of $H$. Clearly $H \Lambda(S) \subset S$. Conversely, let $s \in S$. We have $s=e s$ and $H=e E e \oplus e E(1-e)$. Therefore

$$
H s=H e s=e E e s+e E(1-e) e s=e E e s=R s \subset S .
$$

In other words: $s$ is the image of $e$ in $H$ under the $R$-endomorphism $s$ of $H$ which belongs to $\Lambda(S)$. Accordingly $S \subset H \Lambda(S)$.

(h) $E_{0}(H) \subset E$. It is enough to show that $E$ contains all $R$ endomorphisms $\dot{\phi}$ of $H$ with $r(H \dot{\phi})=1$, because every $R$-endomorphism in $E_{0}(H)$ is a sum of those. If $r(H \dot{\phi})=1$, then by (6.3) we may write $H=\{h\} \oplus K(\dot{\phi})$. Hence to prove $E_{0}(H) \subset E$, we must show 
that to each decomposition $H=\{h\} \oplus H^{\prime}$ and each $y \in H$ there exists an $R$-endomorphism in $E$ which maps $h$ onto $y$ and annihilates $H^{\prime}$. Consider the left ideal $L=\Lambda\left(H^{\prime}\right)$ of $E$. We claim that $L$ is closed in the $J$-adic topology of $E$ and satisfies $q E \cap L=q L$. To this end, let $\left(\alpha_{i}^{\prime}\right)$ be a Cauchy sequence in $L$. This sequence has a limit $\alpha$ in $E$, since $E$ is complete. It follows from (b), (c) and (d) that $\Lambda\left(p^{i} H\right)=$ $J(E)^{i}$ for every positive integer $i$. Therefore, for each $x \in H$, the sequence $\left(w \alpha_{i}^{\prime}\right)$ is Cauchy in the $p$-adic topology of $H$ and converges to $x \alpha^{\prime}$. But each $x \alpha_{i}^{\prime}$ belongs to $H^{\prime}$, and $H^{\prime}$ is a direct summand of $H$. Consequently $x \alpha \in H^{\prime}$ for each $x \in H$, or equivalently, $\alpha \in L$. Thus $L$ is closed. To prove the purity of $L$, note first that $p H=H q$. If now $q \dot{\phi} \in L$, then $H q \dot{\phi}=(p H) \dot{\phi} \subset p H \cap H^{\prime}=p H^{\prime}$. This implies that $H \dot{\phi} \subset H^{\prime}$ because $H$ is torsion-free. Hence $\dot{\phi} \in L$. In other words, $L \cap q E=q L$. Assume next that $R(L)=0$. Then $L$ satisfies the hypothesis of (4), so that $E_{0}$ must be contained in $L$. But this would mean in particular that $H=H H \subset H E_{0} \subset H L \subset H^{\prime}$, contradicting the fact that $H^{\prime}$ is a proper summand of $H$. Hence $R(L) \neq 0$. But by (g) and (6.1) we have $R(L)=P\left(H^{\prime}\right)$. Since $q$ is not a zero-divisor and $\bigcap_{n} E q^{n}=0$, this implies that $P\left(H^{\prime}\right) \not \subset E q$. So pick $\alpha$ in $P\left(H^{\prime}\right)$ with $\alpha \notin E q$. Then $h \alpha \notin J(E)$, since otherwise because of $H^{\prime} \alpha=0$ and (b), $\alpha$ would be divisible by $q$. Now $h \alpha E$ is a right ideal of $E$, contained in $H$ but not in $J(E)$. However, $H$ is minimal nonradical. Hence $h \alpha E=H$. Thus for every $y \in H$ we can find a $\beta \in E$ such that $(h \alpha) \beta=$ $y$, and so $\alpha \beta$ is the desired $R$-endomorphism in $E$ mapping $h$ onto $y$ and annihilating $H^{\prime}$.

(i) $E=E(H)$. Since we just proved that $E_{0}(H) \subset E$, we can now apply the powerful Theorem 6.5. If the rank of $H$ is one, then already $E_{0}(H)=E(H)$. Hence without loss of generality we may assume $r(H) \geqq 2$. In order to show $E=E(H)$ it is by virtue of (8.2) only necessary to show that $E$ contains all idempotent $R$-endomorphisms of $H$. If $\sigma$ is any idempotent of $E(H)$, then we can write $H=H \sigma \oplus$ $K(\sigma)$, where $\sigma$ is the identity on $H \sigma$ and annihilates $K(\sigma)$. Hence we have to prove that to each decomposition $H=H_{1} \oplus H_{2}$ there exists an $R$-endomorphism in $E$ which fixes every element in $H_{1}$ and annihilates $H_{2}$. Consider the two left ideals $L_{1}=\Lambda\left(H_{1}\right)$ and $L_{2}=\Lambda\left(H_{2}\right)$. Clearly $L_{1} \cap L_{2}=0$, and (6.5) tells us that $L_{1}$ and $L_{2}$ are both left annihilators. We wish to show $q E \cap\left(L_{1}+L_{2}\right)=q\left(L_{1}+L_{2}\right)$. The purity of $L_{1}$ and $L_{2}$ themselves is established as under (h). So let $q \alpha \in L_{1}+L_{2}$, say, $q \alpha=\beta_{1}+\beta_{2}$ with $\beta_{i} \in L_{i}$. Pick any $h \in H$. Then $h \beta_{i}=h_{i} \in H_{i}$ and

$$
h_{1}+h_{2}=h\left(\beta_{1}+\beta_{2}\right)=h q \alpha \in H q=p H=p H_{1} \oplus p H_{2} .
$$

Thus $h \beta_{i} \in p H_{i}$, so that $\beta_{i} \in L_{i} \cap \Lambda(p H)=L_{i} \cap J(E)=q L_{i}$. Consequently $\beta_{1}+\beta_{2} \in q\left(L_{1}+L_{2}\right)$, which establishes the validity of $q E \cap$ 
$\left(L_{1}+L_{2}\right)=q\left(L_{1}+L_{2}\right)$. Hence $L_{1}+L_{2}$ satisfies the hypothesis of (5) and is therefore again a left annihilator. Under the lattice isomorphism of (6.5) this left annihilator must correspond to $H_{1} \oplus H_{2}=H$, which means that $L_{1} \oplus L_{2}=E$. Since $E$ possesses an identity element, it follows that there exists an idempotent $\sigma$ in $E$ such that $L_{1}=E \sigma$ and $L_{2}=E(1-\sigma)$. Now, using $(\mathrm{g})$, we have $H_{1}=H \Lambda\left(H_{1}\right)=H E \sigma$ and $H_{2}=H \Lambda\left(H_{2}\right)=H E(1-\sigma)$. Hence $\sigma$ is the identity on $H_{1}$ and annihilates $H_{2}$.

(j) Uniqueness. Suppose that $\alpha$ is a ring isomorphism between $E\left(H_{1}\right)$ and $E\left(H_{2}\right)$, where $H_{i}$ is a reduced complete torsion-free $R_{i}$-module $(i=1,2)$. We know from (7.6) that, if $e_{i}$ is any minimal idempotent in $E\left(H_{i}\right)$, then the $R_{i}$-module $H_{i}$ is isomorphic with the $e_{i} E\left(H_{i}\right) e_{i}$ module $e_{i} E\left(H_{i}\right)$. Since $\alpha$ maps minimal idempotents onto minimal idempotents, it follows that the $R_{1}$-module $H_{1}$ is isomorphic with the $R_{2}$-module $H_{2}$. This completes the proof.

\section{REFERENCES}

1. R. Baer, A unified theory of projective spaces and finite abelian groups, Trans. Amer. Math. Soc., 52 (1942), 283-343.

2. - Linear Algebra and Projective Geometry, Academic Press, New York, 1952.

3. — Dualisierbare Moduln und Praemoduln, Etudes sur les groupes abáliens, Dunod, Paris, 1968.

4. L. Fuchs, Abelian groups, Budapest, 1958.

5. D. K. Harrison, Infinite abelian groups and homological methods, Ann. Math., 69 (1959), 366-391.

6. N. Jacobson, Structure of Rings, Providence, 1956.

7. I. Kaplansky, Infinite Abelian Groups, Univ. of Michigan Press, Ann Arbor, 1954.

8. L. Kulikov, Zur Theorie der abelschen Gruppen von beliebiger Mächtigkeit, Mat. Sbornik, 16 (1945), 129-162.

9. H. Leptin, Zur Theorie der überabzählbaren abelschen p-Gruppen, Abh. Math. Sem. Univ. Hamburg, 24 (1960), 79-90.

10. W. Liebert, Charakterisierung der Endomorphismenringe endlicher abelscher Gruppen, Archiv der Math., 18 (1967), 128-135.

11. - Charakterisierung der Endomorphismenringe beschränkter abelscher Gruppen, Math. Annalen, 174 (1967), 217-232.

12. L Endomorphism rings of abelian p-groups, Etudes sur les groupes abéliens, Dunod, Paris, 1968.

13. E. Matlis, Cotorsion modules, Mem. Amer. Math. Soc., 49 (1964).

14. Y. Utumi, On a theorem on modular lattices, Proc. Japan Acad., 35 (1959), 16-21.

15. R. B. Warfield, Homomorphisms and duality for torsion-free groups, Math. Zeitschr. 107 (1968), 189-200.

16. K. G. Wolfson, An ideal-theoretic characterization of the ring of all linear transformations, Amer. J. Math., 75 (1953), 358-386.

Received September 22, 1969. This research was supported by NSF Grant GP-13286.

New Mexico State University 


\section{PACIFIC JOURNAL OF MATHEMATICS}

\section{EDITORS}

H. SAMELSON

Stanford University

Stanford, California 94305

C. R. Новву

University of Washington

Seattle, Washington 98105
J. DUGundJI

Department of Mathematics

University of Southern California

Los Angeles, California 90007

RICHARD ARENS

University of California

Los Angeles, California 90024

\section{ASSOCIATE EDITORS}
E. F. BECKENBACH
B. H. NeumanN
F. WOLF
K. YOSHIDA

\section{SUPPORTING INSTITUTIONS}
UNIVERSITY OF BRITISH COLUMBIA
CALIFORNIA INSTITUTE OF TECHNOLOGY
UNIVERSITY OF CALIFORNIA
MONTANA STATE UNIVERSITY
UNIVERSITY OF NEVADA
NEW MEXICO STATE UNIVERSITY
OREGON STATE UNIVERSITY
UNIVERSITY OF OREGON
OSAKA UNIVERSITY
UNIVERSITY OF SOUTHERN CALIFORNIA
STANFORD UNIVERSITY
UNIVERSITY OF TOKYO
UNIVERSITY OF UTAH
WASHINGTON STATE UNIVERSITY
UNIVERSITY OF WASHINGTON
AMERICAN MATHEMATICAL SOCIETY CHEVRON RESEARCH CORPORATION TRW SYSTEMS
NAVAL WEAPONS CENTER 


\section{Pacific Journal of Mathematics}

\section{Vol. 37, No. $1 \quad$ January, 1971}

Gregory Frank Bachelis and Haskell Paul Rosenthal, On unconditionally

converging series and biorthogonal systems in a Banach space .........

Richard William Beals, On spectral theory and scattering for elliptic

operators with singular potentials .........................

J. Lennart (John) Berggren, Solvable and supersolvable groups in which every element is conjugate to its inverse ........................ 21

Lindsay Nathan Childs, On covering spaces and Galois extensions ..........

William Jay Davis, David William Dean and Ivan Singer, Multipliers and

unconditional convergence of biorthogonal expansions..............

Leroy John Derr, Triangular matrices with the isoclinal property ............

Paul Erdős, Robert James McEliece and Herbert Taylor, Ramsey bounds for

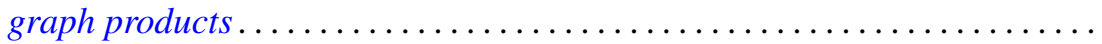

Edward Graham Evans, Jr., On epimorphisms to finitely generated

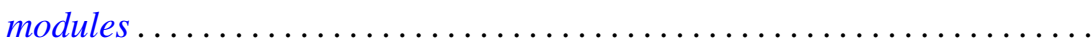

Hector O. Fattorini, The abstract Goursat problem ................. 51

Robert Dutton Fray and David Paul Roselle, Weighted lattice paths .........

Thomas L. Goulding and Augusto H. Ortiz, Structure of semiprime $(p, q)$

radicals ...........................................

E. W. Johnson and J. P. Lediaev, Structure of Noether lattices with join-principal maximal elements ....

David Samuel Kinderlehrer, The regularity of minimal surfaces defined over

slit domains

Alistair H. Lachlan, The transcendental rank of a theory. .

Frank David Lesley, Differentiability of minimal surfaces at the boundary ...

Wolfgang Liebert, Characterization of the endomorphism rings of divisible torsion modules and reduced complete torsion-free modules over complete discrete valuation rings....

Lawrence Carlton Moore, Strictly increasing Riesz norms.

Raymond Moos Redheffer, An inequality for the Hilbert transform ...

James Ted Rogers Jr., Mapping solenoids onto strongly self-entwined,

circle-like continua..........................

Sherman K. Stein, B-sets and planar maps ................... 217

Darrell R. Turnidge, Torsion theories and rings of quotients of Morita

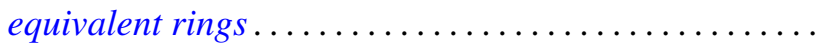

Fred Ustina, The Hausdorff means of double Fourier series and the principle of localization ................................

Stanley Joseph Wertheimer, Quasi-compactness and decompositions for arbitrary relations.

Howard Henry Wicke and John Mays Worrell Jr., On the open continuous images of paracompact $\check{C}$ ech complete spaces... 